\title{
LOCALIZING VIRTUAL FUNDAMENTAL CYCLES FOR SEMI-PERFECT OBSTRUCTION THEORIES
}

\author{
YOUNG-HOON KIEM
}

\begin{abstract}
Recently H.-L. Chang and J. Li generalized the theory of virtual fundamental class to the setting of semi-perfect obstruction theory. A semi-perfect obstruction theory requires only the local existence of a perfect obstruction theory with compatibility conditions. In this paper, we generalize the torus localization of Graber-Pandharipande [17, the cosection localization 21 and their combination [6], to the setting of semi-perfect obstruction theory. As an application, we show that the Jiang-Thomas theory 20] of virtual signed Euler characteristic works without the technical quasi-smoothness assumption from derived algebraic geometry.
\end{abstract}

\section{INTRODUCTION}

The theory of virtual fundamental class was invented in 1995 by LiTian [31] and Behrend-Fantechi [2] in order to provide a rigorous algebrogeometric theory of the Gromov-Witten invariant. Since then, the virtual fundamental class has played a key role in defining important invariants like Donaldson-Thomas and Pandharipande-Thomas invariants, as integrals on the virtual fundamental cycles on suitable moduli spaces. Each DeligneMumford stack $X$ has the intrinsic normal cone $\mathbf{c}_{X}$ canonically embedded into its abelian hull $\mathfrak{N}_{X}=h^{1} / h^{0}\left(\mathbb{L}_{X}^{\vee}\right)$ where $\mathbb{L}_{X}$ denotes the cotangent complex of $X$ (cf. [2]). A perfect obstruction theory $\phi: E \rightarrow \mathbb{L}_{X}$ gives us an embedding of $\mathfrak{N}_{X}$ into the vector bundle stack $\mathcal{E}_{X}=h^{1} / h^{0}\left(E^{\vee}\right)$ and the virtual fundamental class $[X]^{\mathrm{vir}}$ is defined by applying the Gysin map $0 \dot{\mathcal{E}}_{X}$ to $\left[\mathbf{c}_{X}\right]$.

A few effective techniques to handle virtual fundamental classes were discovered during the past two decades, such as the torus localization of Graber-Pandharipande [17], the degeneration method of J. Li [30] and the cosection localization [21. Often combining these techniques turns out to be quite effective. In [6], it was proved that the torus localization works for the cosection localized virtual fundamental classes and this combined localization turned out to be quite useful for the Landau-Ginzburg/CalabiYau correspondence [10].

Date: October 31, 2016.

Partially supported by Samsung Science and Technology Foundation. 
Recently thanks to improved knowledge on derived categories and wall crossing, there arose a demand to handle more moduli spaces of derived category objects on a smooth projective variety. Unfortunately it is often hard to find perfect obstruction theories on moduli spaces of derived category objects and hence virtual fundamental classes were not readily available for them. In [7], H.-L. Chang and J. Li constructed the virtual fundamental class under a weaker condition than the existence of a perfect obstruction theory. They require only the local existence of compatible perfect obstruction theories and it was proved in [7] that these local theories are sufficient to give the virtual fundamental class with desired properties. In fact, this weaker requirement amounts to the theory of tangent-obstruction sheaves of [31]. For many moduli spaces of derived category objects, semi-perfect obstruction theories are easier to construct (cf. [7]) and it was shown in [22] that a critical virtual manifold always has a semi-perfect obstruction theory and hence a virtual fundamental class. The goal of this paper is to generalize and establish the localization theorems in the setting of semi-perfect obstruction theory.

A semi-perfect obstruction theory on a Deligne-Mumford stack $X$ consists of an étale cover $\left\{X_{\alpha} \rightarrow X\right\}$ and perfect obstruction theories

$$
\left\{\phi_{\alpha}: E_{\alpha} \rightarrow \mathbb{L}_{X_{\alpha}}\right\}
$$

of $X_{\alpha}$ satisfying compatibility conditions on the obstruction sheaves and assignments (cf. Definition 2.7). By constructing a cone cycle $\mathcal{C}_{X}$ in the obstruction sheaf $O b_{X}$ and applying the (generalized) Gysin map $00_{\mathcal{F}}^{!}$for the coherent sheaf $\mathcal{F}=O b_{X}$, we obtain the virtual fundamental class $[X]^{\text {vir }}$ with respect to the semi-perfect obstruction theory on $X$. When there is an action of $T=\mathbb{C}^{*}$ and all the local perfect obstruction theories as well as the étale cover $X_{\alpha} \rightarrow X$ are $T$-equivariant, we prove that the usual torus localization formula

$$
[X]^{\text {vir }}=\iota_{*} \frac{[F]^{\text {vir }}}{e\left(N^{\text {vir }}\right)}
$$

holds (cf. Theorem 4.5) where $F$ is the $T$-fixed locus equipped with the induced semi-perfect obstruction theory. The only additional assumption we need is the existence of a global resolution $\left[N_{0} \rightarrow N_{1}\right]$ of the virtual normal bundle $N^{\text {vir }}$ of $F$ by locally free sheaves as in [6].

Compatibility of perfect obstruction theories $\phi_{\alpha}: E_{\alpha} \rightarrow \mathbb{L}_{X_{\alpha}}$ requires that the local obstruction sheaves $O b_{X_{\alpha}}=h^{1}\left(E_{\alpha}^{\vee}\right)$ glue to a sheaf $O b_{X}$ on $X$. If there is a cosection $\sigma: O b_{X} \rightarrow \mathcal{O}_{X}$, we prove (cf. Theorem 3.1) that the virtual fundamental class $[X]^{\text {vir }}$ of $X$ localizes to the zero locus $X(\sigma)$ of $\sigma$, generalizing the cosection localization theorem in [21].

In 6], the authors combined the torus localization with the cosection localization. They proved that the torus localization formula holds for the cosection localized virtual fundamental class if the cosection $\sigma$ is $T$-invariant. 
In 95 , we generalize this combined localization result to the setting of semiperfect obstruction theory (cf. Theorem 5.1).

As an application, we consider the Jiang-Thomas theory of virtual signed Euler characteristic in 20]. For a scheme $X$ equipped with a perfect obstruction theory, Jiang and Thomas collected five natural ways to think of virtual signed Euler characteristic via

(1) K-theory,

(2) torus localization,

(3) Euler characteristic weighted by the Behrend function,

(4) cosection localization and

(5) ordinary Euler characteristic,

and proved that $(1)=(2)$ while $(3)=(4)=(5)$. To make sense of $(2),(3)$ and (4), they constructed the dual obstruction cone $N=\operatorname{Spec}_{X}\left(\operatorname{Sym}\left(O b_{X}\right)\right)$ and proved that $N$ admits a perfect obstruction theory after assuming that $X$ comes from a quasi-smooth derived scheme. We will prove in 86 that $N$ always admits a semi-perfect obstruction theory without any assumption from derived algebraic geometry. Applying the torus localization and cosection localization theorems proved in this paper, we can make sense of (2), (3) and (4) and the Jiang-Thomas theory of virtual signed Euler characteristic is established without the technical assumption.

Here is the layout of the paper. In $₫ 3$, we will prove that the cosection localization of [21] works for semi-perfect obstruction theory. In 4 , we will show that the torus localization of Graber-Pandharipande [17] also works for semi-perfect obstruction theory. In $\$ 5$, we prove that the torus localization works for the cosection localized virtual fundamental class of a semi-perfect obstruction theory. In $\sqrt[6]{6}$, we apply these results to the dual obstruction cone of Jiang-Thomas and show that their theory works without the technical quasi-smoothness assumption from derived algebraic geometry.

All schemes or Deligne-Mumford stacks in this paper are of finite type and defined over the complex number field $\mathbb{C}$. All Chow groups in this paper have coefficients in the rational number field $\mathbb{Q}$.

\section{LOCALIZATION OF VIRTUAL FUNDAMENTAL CYCLES AND SEMI-PERFECT OBSTRUCTION THEORY}

2.1. Localization of virtual fundamental classes. In this subsection, we recall the torus localization of Graber-Pandharipande [17] and the cosection localization for virtual fundamental class (cf. [21]).

Let $X$ be a Deligne-Mumford stack over $\mathbb{C}$. The intrinsic normal cone $\mathbf{c}_{X}$ of $X$ is a cone stack which satisfies $\left[C_{U / V} /\left.T_{V}\right|_{U}\right]=\left.\mathbf{c}_{X}\right|_{U}$ if $U \rightarrow X$ is étale and $U$ is closed in a smooth variety $V$. 
Definition 2.1. 2] A perfect obstruction theory on a Deligne-Mumford stack $X$ is a morphism $\phi: E \rightarrow \mathbb{L}_{X}$ in the derived category $D\left(\mathcal{O}_{X}\right)$ of quasi-coherent $\mathcal{O}_{X}$-modules such that

(1) $E$ is locally isomorphic to a two-term complex $\left[E^{-1} \rightarrow E^{0}\right]$ of locally free sheaves,

(2) $h^{-1}(\phi)$ is surjective and $h^{0}(\phi)$ is an isomorphism.

The perfect obstruction theory is symmetric if there exists an isomorphism $\theta: E \rightarrow E^{\vee}[1]$ with $\theta^{\vee}[1]=\theta$.

Here, $\mathbb{L}_{X}$ denotes the truncated cotangent complex $\tau^{\geq-1} L_{X}$ of $X$.

A perfect obstruction theory $\phi: E \rightarrow \mathbb{L}_{X}$ gives us a vector bundle stack $\mathcal{E}=h^{1} / h^{0}\left(E^{\vee}\right)$ which is locally $E_{1} / E_{0}$ where $E_{i}$ is the dual bundle of $E^{-i}$ for $i=0,1$. Then by [2], the intrinsic normal cone $\mathbf{c}_{X}$ is canonically embedded into the abelian cone stack $h^{1} / h^{0}\left(\mathbb{L}_{X}^{\vee}\right)$ which is embedded into $\mathcal{E}$ by $\phi^{\vee}$. Then the virtual fundamental class of $X$ is defined as

$$
[X]^{\text {vir }}=0 !{ }_{\mathcal{E}}\left[\mathbf{c}_{X}\right]
$$

where $0 !$ is the Gysin map for the vector bundle stack $\mathcal{E}$.

When $X$ admits an action of $T=\mathbb{C}^{*}$ and the perfect obstruction theory $\phi: E \rightarrow \mathbb{L}_{X}$ is $T$-equivariant, the virtual fundamental class of $X$ is localized to the $T$-fixed locus $F$ in $X$ by the formula

$$
[X]^{\text {vir }}=\imath_{*} \frac{[F]^{\text {vir }}}{e\left(N^{\text {vir }}\right)} \in A_{*}^{T}(X) \otimes_{\mathbb{Q}[t]} \mathbb{Q}\left[t, t^{-1}\right]
$$

where $\imath: F \rightarrow X$ is the inclusion and $N^{\text {vir }}$ is the moving part of $\left.E^{\vee}\right|_{F}$ if $N^{\text {vir }}$ admits a global resolution $\left[N_{0} \rightarrow N_{1}\right]$ by locally free sheaves $N_{0}, N_{1}$ on $F$ (cf. [17, 6]).

When the obstruction sheaf $O b_{X}=h^{1}\left(E^{\vee}\right)$ has a cosection $\sigma: O b_{X} \rightarrow$ $\mathcal{O}_{X}$, the virtual fundamental class $[X]^{\text {vir }}$ is localized to the zero locus $X(\sigma)$ of $\sigma$ by [21. The cosection localized virtual fundamental class is obtained in two steps. Firstly we observe that the intrinsic normal cone $\mathbf{c}_{X}$ has support in

$$
\mathcal{E}(\sigma)=\left.\mathcal{E}\right|_{X(\sigma)} \cup \operatorname{ker}\left(\left.\mathcal{E}\right|_{U} \stackrel{\sigma}{\longrightarrow} \mathcal{O}_{U}\right)
$$

where $U=X-X(\sigma)$. Secondly the Gysin map $0 ! A_{\mathcal{E}}: A_{*} \rightarrow A_{*} X$ is localized to a map

$$
0 !_{\mathcal{E}, \mathrm{loc}}: A_{*}(\mathcal{E}(\sigma)) \longrightarrow A_{*}(X(\sigma))
$$

and the cosection localized virtual cycle is defined as

$$
[X]_{\text {loc }}^{\text {vir }}=0 !_{\mathcal{E}, \text { loc }}^{\text {[ }}\left[\mathbf{c}_{X}\right] .
$$

This cosection localized virtual fundamental class satisfies many expected properties such as deformation invariance and

$$
\imath_{*}[X]_{\mathrm{loc}}^{\mathrm{vir}}=[X]^{\mathrm{vir}}
$$

where $\imath: X(\sigma) \rightarrow X$ is the inclusion map. 
Combining the localization techniques is often useful. In [6], the authors proved that the torus localization formula (2.1) holds for the cosection localized virtual cycles, i.e.

$$
[X]_{\mathrm{loc}}^{\mathrm{vir}}=\imath_{*} \frac{[F]_{\mathrm{loc}}^{\mathrm{vir}}}{e\left(N^{\mathrm{vir}}\right)} \in A_{*}^{T}(X(\sigma)) \otimes_{\mathbb{Q}[t]} \mathbb{Q}\left[t, t^{-1}\right]
$$

which turned out to be quite useful.

2.2. Cycles on sheaf stacks. In this section, we recall the notion of cycles on sheaf stacks [7]. Let $X$ be a Deligne-Mumford stack and $\mathcal{F}$ be a coherent sheaf on $X$. Then the groupoid associating sections $s \in \Gamma\left(U, f^{*} \mathcal{F}\right)$ to a morphism $f: U \rightarrow X$ is a stack, called the sheaf stack of $\mathcal{F}$. By abusing the notation, we denote the sheaf stack by the same symbol $\mathcal{F}$.

We will consider the intersection theory on the sheaf stack $\mathcal{F}$.

Definition 2.2. [7, Definition 3.2] (1) A reduced cycle in $\mathcal{F}$ is a substack $B$ of $\mathcal{F}$ such that for any étale morphism $f: U \rightarrow X$ from a scheme $U$ and a surjective homomorphism $\mathcal{V} \rightarrow f^{*} \mathcal{F}$ from a vector bundle $\mathcal{V}$ on $U$, $\tilde{B}=\mathcal{V} \times_{f^{*} \mathcal{F}} f^{*} B \subset \mathcal{V}$ is a Zariski closed reduced subscheme.

(2) Three reduced cycles $B_{1}, B_{2}, B_{3}$ in $\mathcal{F}$ satisfy $B_{3}=B_{1} \cup B_{2}$ if for any étale morphism $f: U \rightarrow X$ from a scheme $U$ and a surjective homomorphism $\mathcal{V} \rightarrow f^{*} \mathcal{F}$ from a vector bundle $\mathcal{V}$ on $U, \tilde{B}_{3}=\tilde{B}_{1} \cup \tilde{B}_{2}$ where $\tilde{B}_{i}=\mathcal{V} \times{ }_{f^{*} \mathcal{F}}$ $f^{*} B_{i}$.

(3) A reduced cycle $B$ in $\mathcal{F}$ is irreducible if it is not the union of two nontrivial reduced cycles $B_{1}$ and $B_{2}$ with $B \neq B_{1}$ and $B \neq B_{2}$.

(4) $A$ prime cycle in $\mathcal{F}$ is an irreducible reduced cycle in $\mathcal{F}$.

(5) The $\mathbb{Q}$-vector space spanned by prime cycles in $\mathcal{F}$ is denoted by $Z_{*}(\mathcal{F})$. Elements of $Z_{*}(\mathcal{F})$ are called cycles on $\mathcal{F}$.

Example 2.3. 1] Let $X$ be a Deligne-Mumford stack equipped with a perfect obstruction theory $E \rightarrow \mathbb{L}_{X}$. Let

$$
\mathbf{c}_{X} \subset \mathcal{E}_{X}=h^{1} / h^{0}\left(E^{\vee}\right)
$$

be the intrinsic normal cone of $X$. Then the obstruction sheaf $O b_{X}=h^{1}\left(E^{\vee}\right)$ is the coarse moduli sheaf of $\mathcal{E}_{X}$ and let

$$
\mathcal{C}_{X} \hookrightarrow O b_{X}
$$

denote the coarse moduli sheaf of $\mathbf{c}_{X}$ so that we have a cartesian diagram

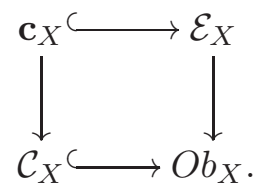

Then for any étale open $f: U \rightarrow X$ and a surjective homomorphism $\mathcal{V} \rightarrow$ $f^{*} O b_{X}$ from a vector bundle $\mathcal{V}$ on $U$, the fiber product

$$
\mathcal{V} \times f^{*} O b_{X} f^{*} \mathcal{C}_{X} \subset \mathcal{V}
$$


gives us a cycle (called the obstruction cone) on the vector bundle $\mathcal{V}$ (cf. [1, $\S 2])$. Therefore $\mathcal{C}_{X} \in Z_{*}\left(O b_{X}\right)$.

It was proved in the proof of [1, Proposition 2.2] that if $V \rightarrow O b_{X}$ is a surjective homomorphism from a vector bundle $V$ on $X$, then the virtual fundamental class is given by

$$
[X]^{\text {vir }}=0 !{ }_{V}^{!}\left[V \times{ }_{O b_{X}} \mathcal{C}_{X}\right] .
$$

In the notation of [7], the right side is $0 ! b_{X}\left(\mathcal{C}_{X}\right)$ so that we have

$$
[X]^{\text {vir }}=0 !_{\mathrm{Ob}_{X}}\left(\mathcal{C}_{X}\right) \text {. }
$$

Let $X$ be a Deligne-Mumford stack and $\mathcal{F}$ be a coherent sheaf on $X$. Let $B \in Z_{*}(\mathcal{F})$ be a prime cycle. Let $\rho: U \rightarrow X$ be an étale morphism with $\rho^{*} B=B \times{ }_{X} U \neq 0 \in Z_{*}\left(\rho^{*} \mathcal{F}\right)$. Pick a vector bundle $F$ on $U$ that admits a surjective homomorphism $F \rightarrow \rho^{*} \mathcal{F}$. Let $\bar{B} \subset U$ be the image of the cycle $\rho^{*} B \times \rho^{*} \mathcal{F} F$ by the projection $F \rightarrow U$. Then the closure $Y$ of $\rho(\bar{B})$ in $X$ is a closed Deligne-Mumford substack. By Chow's lemma [29, 16.6.1], there are a quasi-projective scheme $S$, a generically étale proper surjective morphism $f: S \rightarrow Y$, a locally free sheaf $\mathcal{V}$ and a surjective homomorphism $\mathcal{V} \rightarrow f^{*} \mathcal{F}$. For an open $S^{\prime} \subset S$ such that $\left.f\right|_{S^{\prime}}: S^{\prime} \rightarrow Y$ is étale, let $\tilde{B}$ be the closure in $\mathcal{V}$ of $\left.\mathcal{V}\right|_{S^{\prime}} \times\left.\mathcal{F} B \subset \mathcal{V}\right|_{S^{\prime}}$.

Definition 2.4. The triple $\left(f: S \rightarrow Y, \mathcal{V} \rightarrow f^{*} \mathcal{F}, \tilde{B} \subset \mathcal{V}\right)$ is called a proper representative of $B$.

Let $W \in Z_{*}(\mathcal{F})$ be a prime cycle on a coherent sheaf $\mathcal{F}$ on a DeligneMumford stack $X$. A rational function $h$ on $W$ is an equivalence class of rational functions $h_{f} \in \mathbf{k}(\tilde{W})$ on $\tilde{W}$ for proper representatives $(f, \mathcal{V}, \tilde{W})$ of $W$ where the equivalence relation is defined as follows: Let $\left(f^{\prime}, \mathcal{V}^{\prime}, \tilde{W}^{\prime}\right)$ be another proper representative of $W$ and $h_{f^{\prime}} \in \mathbf{k}\left(\tilde{W}^{\prime}\right)$. Then $h_{f} \sim h_{f^{\prime}}$ if and only if there is a third proper representative $(\hat{f}, \hat{\mathcal{V}}, \hat{W})$ of $W$ that fits into the commutative diagrams
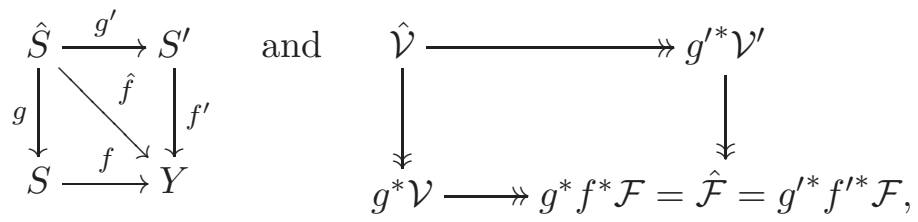

such that the pullbacks of $h_{f}$ and $h_{f^{\prime}}$ to $\hat{W}$ by $g$ and $g^{\prime}$ respectively coincide in $\mathbf{k}(\hat{W})$.

We say a rational function $h$ on a prime cycle $W$ on $\mathcal{F}$ is admissible if for any proper representative $(f, \mathcal{V}, \tilde{W})$, the extension $h_{f}^{\text {nor }}: \tilde{W}^{\text {nor }} \rightarrow \mathbb{P}^{1}$ of $h_{f}$ to the normalization $\tilde{W}^{\text {nor }}$ is constant on the fibers of $\tilde{W}^{\text {nor }} \rightarrow \mathcal{F}$. 
For a prime cycle $W$ on $\mathcal{F}$ and an admissible rational function $h$ on $W$, we write the principal divisor of $h_{f}$ on $\tilde{W}$ as

$$
\partial\left(\tilde{W}, h_{f}\right)=\sum_{i} n_{i} D_{i}
$$

for distinct prime divisors $D_{i}$ on $\tilde{W}$ where $(f, \mathcal{V}, \tilde{W})$ is a proper representative of $W$ and $h_{f}$ is a rational function on $\tilde{W}$ representing $h$. Then the image $W_{i}$ of $D_{i}$ in $\mathcal{F}$ is a prime cycle on $\mathcal{F}$ (cf. [7, §3]). The boundary of $(W, h)$ is now defined as

$$
\partial(W, h)=\sum_{i} n_{i} W_{i} \frac{e_{i}}{\operatorname{deg} f}
$$

where $e_{i}$ is the degree of the morphism $\bar{W}_{i} \rightarrow W_{i}$ where $\bar{W}_{i}$ is the image of $D_{i}$ in $f^{*} \mathcal{F}$.

Definition 2.5. [7] Two cycles $B_{1}$ and $B_{2}$ on a coherent sheaf $\mathcal{F}$ on a Deligne-Mumford stack $X$ are rationally equivalent if $B_{1}-B_{2}$ is a linear combination of cycles of the form $\partial(W, h)$ where $W$ is a prime cycle on $\mathcal{F}$ and $h$ is an admissible rational function on $W . W e$ let $A_{*}(\mathcal{F})$ be the $\mathbb{Q}$-vector space of rational equivalence classes of cycles in $Z_{*}(\mathcal{F})$.

With this preparation, the Gysin map for a coherent sheaf $\mathcal{F}$ is now defined as follows.

Definition 2.6. The Gysin map for $\mathcal{F}$ is defined by

$$
0_{\mathcal{F}}^{!}[B]=\frac{1}{\operatorname{deg} f} f_{*}\left(0_{\mathcal{V}}^{!}[\tilde{B}]\right) \in A_{*} X
$$

where $(f, \mathcal{V}, \tilde{B})$ is a proper representative of $B$.

By [7, Proposition 3.4], $0 !_{\mathcal{F}}[B]$ is independent of the choice of a proper representative of $B$. By [7, Corollary 3.6], the Gysin map $0_{\mathcal{F}}^{!}$preserves rational equivalence and hence induces a map $0 !{ }_{\mathcal{F}}: A_{*}(\mathcal{F}) \longrightarrow A_{*}(X)$.

Suppose there is a homomorphism $\sigma: \mathcal{F} \rightarrow \mathcal{O}_{X}$, called a cosection, of a coherent sheaf $\mathcal{F}$ on a Deligne-Mumford stack $X$. Let $X(\sigma)$ be the zero locus of $\sigma$ whose defining ideal is the image of $\sigma$. Let $U=X-X(\sigma)$ and consider the substack

$$
\mathcal{F}(\sigma)=\left.\mathcal{F}\right|_{X(\sigma)} \cup \operatorname{ker}\left(\sigma:\left.\mathcal{F}\right|_{U} \rightarrow \mathcal{O}_{U}\right) .
$$

A cycle on $\mathcal{F}(\sigma)$ is a cycle on $\mathcal{F}$ which is a substack of $\mathcal{F}(\sigma)$, i.e. a prime cycle $B \in Z_{*}(\mathcal{F})$ belongs to $Z_{*}(\mathcal{F}(\sigma))$ if for a proper representative $(f: S \rightarrow$ $\left.X, \mathcal{V} \rightarrow f^{*} \mathcal{F}, \tilde{B}\right)$ of $B$, we have

$$
\left.\tilde{B} \subset \mathcal{V}\right|_{S \times_{X} X(\sigma)} \cup \operatorname{ker}\left(\tilde{\sigma}: \mathcal{V} \rightarrow \mathcal{O}_{S}\right)
$$

where $\tilde{\sigma}: \mathcal{V} \rightarrow \mathcal{O}_{S}$ is the composition of the surjective homomorphism $\mathcal{V} \rightarrow f^{*} \mathcal{F}$ with $f^{*} \sigma: f^{*} \mathcal{F} \rightarrow \mathcal{O}_{S}$. We say two cycles $B_{1}$ and $B_{2}$ on $\mathcal{F}(\sigma)$ are rationally equivalent if $B_{1}-B_{2}$ is a linear combination of cycles of the 
form $\partial(W, h)$ where $W$ is a prime cycle on $\mathcal{F}(\sigma)$ and $h$ is an admissible rational function on $W$. We let $A_{*}(\mathcal{F}(\sigma))$ be the $\mathbb{Q}$-vector space of rational equivalence classes of cycles on $\mathcal{F}(\sigma)$.

2.3. Semi-perfect obstruction theory. A semi-perfect obstruction theory is a generalization of a perfect obstruction theory which still gives rise to a virtual fundamental class.

Definition 2.7. 7] A semi-perfect obstruction theory on a Deligne-Mumford stack $X$ consists of an étale cover $\left\{X_{\alpha} \rightarrow X\right\}$ and a perfect obstruction theory $\phi_{\alpha}: E_{\alpha} \rightarrow \mathbb{L}_{X_{\alpha}}$ for each $\alpha$ such that

(1) there are isomorphisms $\psi_{\alpha \beta}:\left.\left.h^{1}\left(E_{\alpha}^{\vee}\right)\right|_{X_{\alpha \beta}} \rightarrow h^{1}\left(E_{\beta}^{\vee}\right)\right|_{X_{\alpha \beta}}$ for pairs of indices $\alpha, \beta$, that glue $\left\{h^{1}\left(E_{\alpha}^{\vee}\right)\right\}$ to a sheaf $O b_{X}$ on $X$ and that

(2) the perfect obstruction theories $\left.E_{\alpha}\right|_{X_{\alpha \beta}}$ and $\left.E_{\beta}\right|_{X_{\alpha \beta}}$ give the same obstruction assignment via $\psi_{\alpha \beta}$ for pairs of indices $\alpha, \beta$.

Here $X_{\alpha \beta}=X_{\alpha} \times_{X} X_{\beta}$ as usual.

The second condition in Definition 2.7 means the following.

Definition 2.8. Let $x \in U$ be a closed point of a Deligne-Mumford stack. $A n$ infinitesimal lifting problem on $U$ consists of an extension $0 \rightarrow I \rightarrow B \rightarrow$ $\bar{B} \rightarrow 0$ of Artin local rings with $I \cdot m_{B}=0$ and a morphism $\bar{g}: \operatorname{Spec} \bar{B} \rightarrow U$ that sends the closed point of $\operatorname{Spec} \bar{B}$ to $x$.

If $\phi: E \rightarrow \mathbb{L}_{U}$ is a perfect obstruction theory on $U$, letting $\bar{\Delta}=\operatorname{Spec} \bar{B}$ and $\Delta=\operatorname{Spec} B$, the natural morphism $\mathbb{L}_{\bar{\Delta}} \rightarrow \mathbb{L}_{\bar{\Delta} / \Delta}=I[1]$ composed with $\phi$ gives us

$$
\bar{g}^{*} E \stackrel{\bar{g}^{*} \phi}{\longrightarrow} \bar{g}^{*} \mathbb{L}_{X} \stackrel{\bar{g}}{\longrightarrow} \mathbb{L}_{\bar{\Delta}} \longrightarrow \mathbb{L}_{\bar{\Delta} / \Delta}=I[1]
$$

which is the obstruction class

$$
o b_{U}(\phi, \bar{g}, B, \bar{B})=\left(\bar{g}^{*} E \rightarrow I[1]\right) \in \operatorname{Ext}^{1}\left(\bar{g}^{*} E, I\right)=\left.I \otimes_{\mathbb{C}} h^{1}\left(E^{\vee}\right)\right|_{x} .
$$

Two obstruction theories $\phi: E \rightarrow \mathbb{L}_{U}$ and $\phi^{\prime}: E^{\prime} \rightarrow \mathbb{L}_{U}$ give the same obstruction assignment via an isomorphism $\psi: h^{1}\left(E^{\vee}\right) \stackrel{\cong}{\longrightarrow} h^{1}\left(E^{\prime \vee}\right)$ of obstruction sheaves if

$$
o b_{U}\left(\phi^{\prime}, \bar{g}, B, \bar{B}\right)=\left.\psi\left(o b_{U}(\phi, \bar{g}, B, \bar{B})\right) \in I \otimes_{\mathbb{C}} h^{1}\left(E^{\prime \vee}\right)\right|_{x}
$$

for any infinitesimal lifting problem $(\bar{g}, B, \bar{B})$.

It was proved in [7] that the conditions in Definition 2.7 guarantee that the images of

$$
\mathbf{c}_{X_{\alpha}} \hookrightarrow \mathcal{E}_{\alpha} \longrightarrow h^{1}\left(E_{\alpha}^{\vee}\right)=O b_{X_{\alpha}}
$$

glue to a cone $\mathcal{C}_{X} \subset O b_{X}$ by the following.

Proposition 2.9. 7] Let $\mathfrak{N}_{X}=h^{1} / h^{0}\left(\mathbb{L}_{X}^{\vee}\right)$ denote the abelian hull of the intrinsic normal cone $\mathbf{c}_{X}$ of $X$. Then the morphisms

$$
\eta_{\alpha}:\left.\mathfrak{N}_{X}\right|_{X_{\alpha}}=h^{1} / h^{0}\left(\mathbb{L}_{X_{\alpha}}^{\vee}\right) \longrightarrow h^{1} / h^{0}\left(E_{\alpha}^{\vee}\right) \longrightarrow h^{1}\left(E_{\alpha}^{\vee}\right)=\left.O b_{X}\right|_{X_{\alpha}}
$$

glue to a morphism $\eta: \mathfrak{N}_{X} \rightarrow O b_{X}$. Moreover, if $A \in Z_{*}\left(\mathfrak{N}_{X}\right)$, then $\left\{\left(\eta_{\alpha}\right)_{*}\left(\left.A\right|_{X_{\alpha}}\right)\right\}$ glue to a cycle $\eta_{*}[A] \in Z_{*}\left(O b_{X}\right)$. 
Applying the Gysin map

$$
0 !_{\mathcal{F}}: A_{*}(\mathcal{F}) \longrightarrow A_{*}(X), \quad \mathcal{F}=O b_{X}
$$

to the image $\mathcal{C}_{X} \in Z_{*}\left(O b_{X}\right)$ of the intrinsic normal cone $\mathbf{c}_{X} \in Z_{*}\left(\mathfrak{N}_{X}\right)$, we get the virtual fundamental class $[X]^{\text {vir }}$ of $X$ for the semi-perfect obstruction theory.

We will show below that the torus localization of Graber-Pandharipande [17] (cf. \$4) and the cosection localization [21] (cf. \$3) as well as their combination (cf. \$5) work for semi-perfect obstruction theories.

\section{Cosection localizAtion For SEMi-PERfECt OBStRuCtion theORY}

In this section, we generalize the cosection localization principle in 21 to the setting of semi-perfect obstruction theory in [7.

Let $X$ be a Deligne-Mumford stack over $\mathbb{C}$ equipped with a semi-perfect obstruction theory

$$
\left(X_{\alpha} \rightarrow X, \quad \phi_{\alpha}: E_{\alpha} \rightarrow \mathbb{L}_{X_{\alpha}}\right) .
$$

Let $O b_{X}$ denote the obstruction sheaf, the gluing of $h^{1}\left(E_{\alpha}^{\vee}\right)$. Let

$$
\sigma: O b_{X} \longrightarrow \mathcal{O}_{X}
$$

be a homomorphism, called a cosection of the obstruction sheaf. Let $X(\sigma)$ be the closed substack (zero locus of $\sigma$ ) of $X$ defined by the ideal $\sigma\left(O b_{X}\right) \subset \mathcal{O}_{X}$ and let $U=X-X(\sigma)$ be the open substack over which $\sigma$ is surjective.

The goal of this section is to prove the following generalization of [21, Theorem 1.1].

Theorem 3.1. (Cosection localization for semi-perfect obstruction theory) Let $X$ be a Deligne-Mumford stack equipped with a semi-perfect obstruction theory. Suppose the obstruction sheaf $O b_{X}$ admits a cosection $\sigma: O b_{X} \rightarrow$ $\mathcal{O}_{X}$. Let $X(\sigma)$ be the zero locus of $\sigma$ and $\imath: X(\sigma) \rightarrow X$ denote the inclusion. Then there exists a localized virtual fundamental class

$$
[X]_{\mathrm{loc}}^{\mathrm{vir}} \in A_{*}(X(\sigma))
$$

satisfying $\imath_{*}[X]_{\mathrm{loc}}^{\mathrm{vir}}=[X]^{\mathrm{vir}} \in A_{*}(X)$.

Since $\left.O b_{X}\right|_{X_{\alpha}}=h^{1}\left(E_{\alpha}^{\vee}\right)$, we have a canonical morphism of stacks

$$
\mathcal{E}_{\alpha}=h^{1} / h^{0}\left(E_{\alpha}^{\vee}\right) \longrightarrow h^{1}\left(E_{\alpha}^{\vee}\right)=\left.O b_{X}\right|_{X_{\alpha}} .
$$

Let $U=X-X(\sigma)$ and $U_{\alpha}=U \times_{X} X_{\alpha}$. The cosection $\sigma$ in (3.2) together with (3.3) induces a morphism

$$
\bar{\sigma}_{\alpha}:\left.\mathcal{E}_{\alpha}\right|_{U_{\alpha}} \longrightarrow O b_{U_{\alpha}} \stackrel{\sigma}{\longrightarrow} \mathcal{O}_{U_{\alpha}} .
$$

Then by [21, Proposition 4.3], $\left[\mathbf{c}_{X_{\alpha}}\right] \in Z_{*}\left(\mathcal{E}_{\alpha}(\sigma)\right)$ where

$$
\mathcal{E}_{\alpha}(\sigma)=\left.\mathcal{E}_{\alpha}\right|_{X_{\alpha}(\sigma)} \cup \operatorname{ker}\left[\bar{\sigma}_{\alpha}:\left.\mathcal{E}_{\alpha}\right|_{U_{\alpha}} \rightarrow \mathcal{O}_{U_{\alpha}}\right], \quad X_{\alpha}(\sigma)=X_{\alpha} \times_{X} X(\sigma) .
$$

Hence the image of $\left[\mathbf{c}_{X_{\alpha}}\right]$ by $\mathcal{E}_{\alpha} \rightarrow O b_{X_{\alpha}}$ lies in $Z_{*}\left(O b_{X_{\alpha}}(\sigma)\right)$ where

$$
O b_{X_{\alpha}}(\sigma)=\left.O b_{X_{\alpha}}\right|_{X_{\alpha}(\sigma)} \cup \operatorname{ker}\left[\sigma_{\alpha}: O b_{U_{\alpha}} \rightarrow \mathcal{O}_{U_{\alpha}}\right] .
$$


Since $\mathcal{C}_{X}$ is the gluing of the images of $\left[\mathbf{c}_{X_{\alpha}}\right]$ and $O b_{X}$ is the gluing of $O b_{X_{\alpha}}$ while $\sigma$ is the gluing of $\sigma_{\alpha}$, letting

$$
O b_{X}(\sigma)=\left.O b_{X}\right|_{X(\sigma)} \cup \operatorname{ker}\left[\sigma_{U}: O b_{U} \rightarrow \mathcal{O}_{U}\right],
$$

we find that

$$
\left[\mathcal{C}_{X}\right] \in A_{*}\left(O b_{X}(\sigma)\right)
$$

Next, we generalize the cosection localized Gysin map (cf. 21, Proposition 1.3]).

Proposition 3.2. Let $\mathcal{F}$ be a coherent sheaf on a Deligne-Mumford stack $X$ and let $\sigma: \mathcal{F} \rightarrow \mathcal{O}_{X}$ be a nonzero cosection. Let $X(\sigma)$ denote the zero locus of $\sigma$ and $U=X-X(\sigma)$ so that $\sigma$ is surjective over $U$. Let $\mathcal{F}(\sigma)=$ $\left.\mathcal{F}\right|_{X(\sigma)} \cup \operatorname{ker}\left[\sigma_{U}:\left.\mathcal{F}\right|_{U} \rightarrow \mathcal{O}_{U}\right]$. Then there is a homomorphism

$$
0 !_{\mathcal{F}, \sigma}: A_{*}(\mathcal{F}(\sigma)) \longrightarrow A_{*}(X(\sigma))
$$

which we call the localized Gysin map, such that

$$
\imath_{*} \circ 00_{\mathcal{F}, \sigma}=0 !_{\mathcal{F}} \circ \tilde{\imath}_{*}: A_{*} \mathcal{F}(\sigma) \longrightarrow A_{*}(X)
$$

where $\imath: X(\sigma) \rightarrow X$ and $\tilde{\imath}: \mathcal{F}(\sigma) \rightarrow \mathcal{F}$ denote the inclusions and $0 !$ is the Gysin map in Definition 2.6.

Proof. Let $B \in Z_{*}(\mathcal{F}(\sigma))$ be a prime cycle. If $B \in Z_{*}\left(\left.\mathcal{F}\right|_{X(\sigma)}\right)$, then we let

$$
0 !_{\mathcal{F}, \sigma}[B]:=0 !_{\left.\mathcal{F}\right|_{X(\sigma)}}[B] \in A_{*}(X(\sigma))
$$

where $0 !_{\left.\mathcal{F}\right|_{X(\sigma)}}$ is the Gysin map of the sheaf $\left.\mathcal{F}\right|_{X(\sigma)}$ on $X(\sigma)$ defined in [7, $\S 3]$. From now on, we let the prime cycle $B$ satisfy $B \notin Z_{*}\left(\left.\mathcal{F}\right|_{X(\sigma)}\right)$.

Let $\pi: \mathcal{F} \rightarrow X$ denote the projection. Let $\rho: X^{\#} \rightarrow X$ be the blowup along $X(\sigma)$, i.e. along the ideal $\mathcal{I}_{\sigma}=\sigma(\mathcal{F}) \subset \mathcal{O}_{X}$ so that we have a surjective homomorphism

$$
\sigma^{\#}: \mathcal{F}^{\#}=\rho^{*} \mathcal{F} \longrightarrow \mathcal{O}_{X}(D)
$$

where $D$ is a Cartier divisor on $\widetilde{X}$ with support $|D|$ such that $\mathcal{O}_{X \#}(D)=$ $\rho^{-1} \mathcal{I}_{\sigma} \cdot \mathcal{O}_{X^{\#}}$ and $\rho(|D|) \subset X(\sigma)$.

For a proper representative $\left(f: S \rightarrow X, \mathcal{V} \rightarrow f^{*} \mathcal{F}, \tilde{B}\right)$, let $S^{\#}$ be the blowup of $S$ along $S(\sigma)=X(\sigma) \times_{X} S$, so that we have a commutative diagram

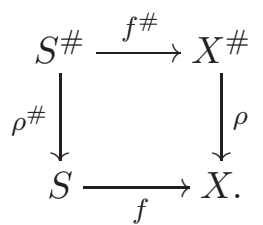


Let $-D_{S}$ denote the exceptional divisor of $\rho^{\#}$. Let $\tilde{\mathcal{F}}=f^{*} \mathcal{F}, \mathcal{V}^{\#}=\left(\rho^{\#}\right)^{*} \mathcal{V}$ and $\tilde{\mathcal{F}}^{\#}=\left(\rho \circ f^{\#}\right)^{*} \mathcal{F}=\left(f \circ \rho^{\#}\right)^{*} \mathcal{F}$. Let $\tilde{B}^{\#} \subset \mathcal{V}^{\#}$ be the proper transform of $\tilde{B} \subset \mathcal{V}$. The cosection $\sigma: \mathcal{F} \rightarrow \mathcal{O}_{X}$ and (3.8) induce a surjective homomorphism

$$
\tilde{\sigma}^{\#}: \mathcal{V}^{\#} \rightarrow \tilde{\mathcal{F}}^{\#} \rightarrow \mathcal{O}_{S \#}\left(D_{S}\right)
$$

We now define

$$
0_{\mathcal{F}, \sigma}^{!}[B]:=\frac{1}{\operatorname{deg} f} f_{*}^{\prime}\left(\rho^{\#}\right)_{*}^{\prime}\left(D_{S} \cdot 0_{\text {ker } \tilde{\sigma} \#}^{!}\left[\tilde{B}^{\#}\right]\right) \in A_{*}(X(\sigma)),
$$

where $f^{\prime}: S(\sigma) \rightarrow X(\sigma)$ is the restriction of $f$ to $S(\sigma)$ and $\left(\rho^{\#}\right)^{\prime}: D_{S} \rightarrow$ $S(\sigma)$ is the restriction of $\rho^{\#}$ to $D_{S}$. Here $D$. denotes the intersection with the divisor $D$ (cf. [14, Chapter 2]). By the definition of the localized Gysin map for vector bundles in [21], we have

$$
0_{\mathcal{V}, \sigma}^{!}[\tilde{B}]=\left(\rho^{\#}\right)_{*}^{\prime}\left(D_{S} \cdot 0_{\mathrm{ker} \tilde{\sigma} \#}^{!}\left[\tilde{B}^{\#}\right]\right) \in A_{*}(S(\sigma))
$$

so that we have

$$
0_{\mathcal{F}, \sigma}^{!}[B]=\frac{1}{\operatorname{deg} f} f_{*}^{\prime} 0_{\mathcal{V}, \sigma}^{\prime}[\tilde{B}] \in A_{*}(X(\sigma)) .
$$

It was also proved in $[21, \S 2]$ that $0_{\dot{\mathcal{V}}, \sigma}^{!}$preserves rational equivalence.

By the usual argument used in [21, §2] or [7, §3], it is straightforward to see that $0 !_{\mathcal{F}, \sigma}[B]$ is independent of the choice of a proper representative of $B$. Indeed, if we have two proper representatives $\left(f_{1}, \mathcal{V}_{1}, \tilde{B}_{1}\right)$ and $\left(f_{2}, \mathcal{V}_{2}, \tilde{B}_{2}\right)$, we can choose a third proper representative $(\hat{f}, \hat{\mathcal{V}}, \hat{B})$ dominating the previous two. Then we can compare the cycles defined by the right side of (3.9) on $\hat{\mathcal{V}}$. We omit the detail here.

By extending (3.7) and (3.9) linearly, we obtain a homomorphism

$$
0 !_{\mathcal{F}, \sigma}: Z_{*}(\mathcal{F}(\sigma)) \longrightarrow A_{*}(X(\sigma)) .
$$

To prove that (3.12) preserves rational equivalence, let $W$ be a prime cycle on $\mathcal{F}(\sigma)$ and $h$ be an admissible rational function on $W$. When $W \in$ $Z_{*}\left(\left.\mathcal{F}\right|_{X(\sigma)}\right)$, this fact was proved in [7]. So we may assume $W \notin Z_{*}\left(\left.\mathcal{F}\right|_{X(\sigma)}\right)$. For a proper representative $\left(f: S \rightarrow X, \mathcal{V} \rightarrow f^{*} \mathcal{F}, \tilde{W}\right)$ of $W$ and a rational function $h_{f}$ on $\tilde{W} \subset \mathcal{V}$ representing $h$, we write the principal divisor of $h_{f}$ on $\tilde{W}$ as $\partial\left(\tilde{W}, h_{f}\right)=\sum n_{i} D_{i}$ where $D_{i}$ are distinct prime divisors. As we reviewed in 22.2 the principal divisor $\partial(W, h)$ of $h$ on $W$ is defined as $\partial(W, h)=\sum n_{i} W_{i} e_{i} / \operatorname{deg} f$ where $W_{i}$ is the image of $D_{i}$ in $\mathcal{F}$ and $e_{i}$ is the degree of the morphism $\bar{W}_{i} \rightarrow W_{i}$ where $\bar{W}_{i}$ is the image of $D_{i}$ in $f^{*} \mathcal{F}$. By [7, $\S 3]$, if $\left(f_{i}, \mathcal{V}_{i}, \tilde{W}_{i}\right)$ is a proper representative of $W_{i}$, then

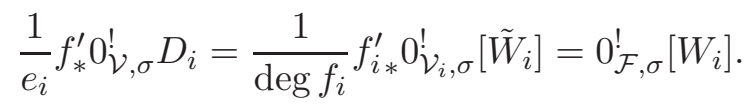

Therefore we have

$$
0_{\mathcal{F}, \sigma}^{!} \partial(W, h)=0_{\mathcal{F}, \sigma}^{!} \sum n_{i} W_{i} \cdot \frac{e_{i}}{\operatorname{deg} f}=\frac{1}{\operatorname{deg} f} \sum n_{i} \cdot e_{i} \cdot 0_{\mathcal{F}, \sigma}^{!} W_{i}
$$




$$
=\frac{1}{\operatorname{deg} f} \sum n_{i} f_{*}^{\prime} 0_{\dot{\mathcal{V}}, \sigma}^{!} D_{i}=\frac{1}{\operatorname{deg} f} f_{*}^{\prime} 0_{\mathcal{V}, \sigma}^{!} \sum n_{i} D_{i}=\frac{1}{\operatorname{deg} f} f_{*}^{\prime} 0_{\dot{\mathcal{V}}, \sigma}^{\prime} \partial\left(\tilde{W}, h_{f}\right) .
$$

Since $0 !_{\mathcal{V}, \sigma}^{!}\left(\partial\left(\tilde{W}, h_{f}\right)\right)=0$ by $[21$, $\S 2]$, we have

$$
0_{\mathcal{F}, \sigma}^{!} \partial(W, h)=0
$$

as desired.

Finally we prove (3.6). For a prime cycle $B \in Z_{*}\left(\left.\mathcal{F}\right|_{X(\sigma)}\right)$ and a proper representative $\left(f: S \rightarrow X, \mathcal{V} \rightarrow f^{*} \mathcal{F}, \tilde{B}\right)$, we have a commutative square

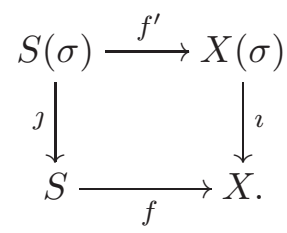

Since $\jmath_{*} 0_{\mathcal{V}, \sigma}^{!}[\tilde{B}]=0_{\mathcal{V}}^{!}[\tilde{B}]$ by $[21, \S 2],(3.6)$ follows from

$\imath_{*} 0_{\mathcal{F}, \sigma}^{!}[B]=\frac{1}{\operatorname{deg} f} \imath_{*} f_{*}^{\prime} 0 \dot{\mathcal{V}}, \sigma^{\prime}[\tilde{B}]=\frac{1}{\operatorname{deg} f} f_{*} \jmath_{*} 0 \dot{\mathcal{V}}, \sigma^{\prime}[\tilde{B}]=\frac{1}{\operatorname{deg} f} f_{*} 0_{\mathcal{V}}^{!}[\tilde{B}]=0_{\mathcal{F}}^{!}[B]$.

Note that our definition (3.11) of the localized Gysin map $00_{\mathcal{F}, \sigma}$ is the same as Definition 2.6 with the ordinary Gysin map $0 !$ replaced by the localized Gysin map $0{ }_{\mathcal{V}, \text { loc }}^{!}=00_{\mathcal{V}, \sigma}^{!}$defined in [21, §2].

Proof of Theorem [3.1. By (3.4), we have the virtual normal cone $\left[\mathcal{C}_{X}\right] \in$ $A_{*}\left(O b_{X}(\sigma)\right)$. Using $0_{\mathcal{F}, \sigma}^{!}$in (3.5) with $\mathcal{F}=O b_{X}$, we define

$$
[X]_{\mathrm{loc}}^{\mathrm{vir}}=00_{O b_{X}, \sigma}^{!}\left[\mathcal{C}_{X}\right] \in A_{*}(X(\sigma)) .
$$

Since $[X]^{\mathrm{vir}}=0_{\mathrm{Ob}_{X}}^{!}\left[\mathcal{C}_{X}\right]$ by definition,

$$
\imath_{*}[X]_{\text {loc }}^{\text {vir }}=\imath_{*} 0 !_{O b_{X}, \sigma}\left[\mathcal{C}_{X}\right]=0 !{ }_{O b_{X}}\left[\mathcal{C}_{X}\right]=[X]^{\text {vir }} \in A_{*}(X)
$$

by $(3.6)$

As in the case of ordinary virtual fundamental class, the localized virtual cycle $[X]_{\text {loc }}^{\text {vir }}$ for a semi-perfect obstruction theory remains constant under deformation. Let $X$ be a Deligne-Mumford stack over $\mathbb{C}$. Let $\left\{X_{\alpha} \rightarrow X\right\}$ be an étale cover and let $\left\{\phi_{\alpha}: E_{\alpha} \rightarrow \mathbb{L}_{X_{\alpha}}\right\}$ be a semi-perfect obstruction theory for $X$. Let $\mathfrak{X}$ be a Deligne-Mumford stack together with a morphism $\pi: \mathfrak{X} \rightarrow T$ to a pointed smooth curve $0 \in T$.

Suppose $X=\mathfrak{X} \times_{T}\{0\}$ and there is a semi-perfect obstruction theory

$$
\left\{\mathfrak{X}_{\alpha} \rightarrow \mathfrak{X}\right\}, \quad\left\{\psi_{\alpha}: F_{\alpha} \rightarrow \mathbb{L}_{\mathfrak{X}_{\alpha}}\right\}, \quad X_{\alpha}=\mathfrak{X}_{\alpha} \times_{T}\{0\}
$$


for $\mathfrak{X}$ together with commutative diagrams

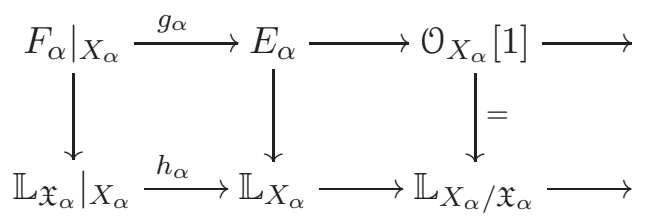

of distinguished triangles. We further assume that the homomorphisms

$$
h^{1}\left(g_{\alpha}^{\vee}\right): h^{1}\left(E_{\alpha}^{\vee}\right) \longrightarrow h^{1}\left(\left.F_{\alpha}\right|_{X_{\alpha}} ^{\vee}\right)=\left.h^{1}\left(F_{\alpha}^{\vee}\right)\right|_{X_{\alpha}}
$$

glue to a homomorphism

$$
\left.O b_{X} \longrightarrow O b_{\mathfrak{X}}\right|_{X}
$$

Let us suppose that there is a homomorphism

$$
\tilde{\sigma}: O b_{\mathfrak{X}} \longrightarrow \mathcal{O}_{\mathfrak{X}}
$$

and let $\sigma: O b_{X} \rightarrow O b_{\mathfrak{X}} \mid X \stackrel{\left.\tilde{\sigma}\right|_{X}}{\longrightarrow} \mathcal{O}_{X}$ denote the composition of (3.17) and (3.16) , restricted to $X$. Let $\mathfrak{X}(\tilde{\sigma})$ be the zero locus of $\tilde{\sigma}$ and $X(\sigma)$ be the zero locus of $\sigma$ so that we have a fiber square

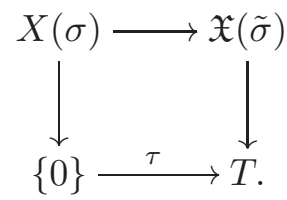

Let

$$
\tau^{!}: A_{*}(\mathfrak{X}(\tilde{\sigma})) \longrightarrow A_{*}(X(\sigma))
$$

denote the Gysin map.

Theorem 3.3. $\tau^{!}[\mathfrak{X}]_{\mathrm{loc}}^{\mathrm{vir}}=[X]_{\mathrm{loc}}^{\mathrm{vir}}$.

By pushing the cycles to obstruction sheaves via the natural transformation $h^{1} / h^{0} \rightarrow h^{1}$, the proof is a straightforward adaptation of the proof of [21. Theorem 5.2] and so we omit it. See also the proof of [7, Proposition $3.8]$.

\section{TORUS LOCALIZATION FOR SEMI-PERFECT OBSTRUCTION THEORY}

In this section, we generalize the torus localization formula in [17 to the setting of semi-perfect obstruction theory. See 6] for the torus localization formula for cosection localized virtual fundamental class.

Let $X$ be a Deligne-Mumford stack acted on by a torus $T=\mathbb{C}^{*}$. Let $F$ be the $T$-fixed locus, locally defined by $\operatorname{Spec} A / A^{m v}$ on an equivariant étale $\operatorname{Spec} A \rightarrow X$, where $A^{m v}$ denotes the ideal generated by weight spaces corresponding to nontrivial $T$-weights. Let

$$
\imath: F \hookrightarrow X
$$

denote the inclusion map. 
Definition 4.1. A T-equivariant semi-perfect obstruction theory on $X$ consists of

(1) a T-equivariant étale open cover $\left\{X_{\alpha} \rightarrow X\right\}$ of $X$;

(2) an object $E_{\alpha} \in D\left(\left[X_{\alpha} / T\right]\right)$ and a morphism

$$
\phi_{\alpha}: E_{\alpha} \rightarrow \mathbb{L}_{X_{\alpha}} \text { in } D\left(\left[X_{\alpha} / T\right]\right)
$$

which is a perfect obstruction theory on $X_{\alpha}$.

Here $D\left(\left[X_{\alpha} / T\right]\right)$ denotes the derived category of $T$-equivariant quasicoherent sheaves on $X_{\alpha}$.

The $T$-fixed locus $F$ admits an induced semi-perfect obstruction theory. Indeed, the $T$-equivariant perfect complex $E_{\alpha} \in D^{b}\left(\left[X_{\alpha} / T\right]\right)$ restricted to $F_{\alpha}=F \times_{X} X_{\alpha}$ decomposes into a direct sum

$$
\left.E_{\alpha}\right|_{F_{\alpha}}=\left.\left.E_{\alpha}\right|_{F_{\alpha}} ^{f i x} \oplus E_{\alpha}\right|_{F_{\alpha}} ^{m v}
$$

of the $T$-fixed part and moving part. Moreover $\phi_{\alpha}: E_{\alpha} \rightarrow \mathbb{L}_{X_{\alpha}}$ also decomposes into the sum of

$$
\phi_{\alpha}^{f i x}:\left.\left.E_{\alpha}\right|_{F_{\alpha}} ^{f i x} \rightarrow \mathbb{L}_{X_{\alpha}}\right|_{F_{\alpha}} ^{f i x} \quad \text { and } \quad \phi_{\alpha}^{m v}:\left.\left.E_{\alpha}\right|_{F_{\alpha}} ^{m v} \rightarrow \mathbb{L}_{X_{\alpha}}\right|_{F_{\alpha}} ^{m v} .
$$

Since $F_{\alpha}$ is $T$-fixed, the morphism $\left.\mathbb{L}_{X_{\alpha}}\right|_{F_{\alpha}} \rightarrow \mathbb{L}_{F_{\alpha}}$ factors through

$$
\left.\mathbb{L}_{X_{\alpha}}\right|_{F_{\alpha}} ^{f i x} \longrightarrow \mathbb{L}_{F_{\alpha}} \text {. }
$$

Let

$$
E_{\alpha}^{F}:=\left.E_{\alpha}\right|_{F_{\alpha}} ^{f i x}, \quad N_{\alpha}^{\mathrm{vir}}:=\left(\left.E_{\alpha}\right|_{F_{\alpha}} ^{m v}\right)^{\vee} .
$$

Lemma 4.2. The composition of (4.2) and (4.3) gives us a perfect obstruction theory

$$
\phi_{\alpha}^{F}: E_{\alpha}^{F} \longrightarrow \mathbb{L}_{F_{\alpha}} .
$$

These perfect obstruction theories $\left\{\phi_{\alpha}^{F}: E_{\alpha}^{F} \rightarrow \mathbb{L}_{F_{\alpha}}\right\}$ form a semi-perfect obstruction theory on $F$, with respect to the étale cover $\left\{F_{\alpha} \rightarrow F\right\}$.

Proof. The first statement follows from [6, Lemma 3.3]. For the second, observe that we have a decomposition

$$
\left.O b_{X_{\alpha}}\right|_{F_{\alpha}}=\left.h^{1}\left(E_{\alpha}^{\vee}\right)\right|_{F_{\alpha}}=h^{1}\left(\left.E_{\alpha}\right|_{F_{\alpha}} ^{\vee}\right)=h^{1}\left(E_{\alpha}^{F^{\vee}}\right) \oplus h^{1}\left(N_{\alpha}^{\text {vir }}\right)
$$

into the direct sum of the $T$-fixed and moving parts. Since $\left\{O b_{X_{\alpha}}\right\}$ glue to $O b_{X}$ by assumption, $\left\{\left.O b_{X_{\alpha}}\right|_{F_{\alpha}}\right\}$ glue to $\left.O b_{X}\right|_{F}$ and hence

$$
\left\{O b_{F_{\alpha}}:=h^{1}\left(\left(E_{\alpha}^{F}\right)^{\vee}\right)\right\}
$$

glue to

$$
O b_{F}:=\left.O b_{X}\right|_{F} ^{f i x} .
$$
lem

By composing with the inclusion $F_{\alpha} \hookrightarrow X_{\alpha}$, an infinitesimal lifting prob-

$$
\bar{g}: \operatorname{Spec} \bar{B} \longrightarrow F_{\alpha}, \quad \bar{B}=B / I, \quad I \cdot m_{B}=0
$$


for $F_{\alpha}$ with obstruction class

$$
\left.o b_{F_{\alpha}}\left(\phi_{\alpha}^{F}, \bar{g}, B, \bar{B}\right) \in I \otimes_{\mathbb{C}} O b_{X_{\alpha}}\right|_{x} ^{f i x}=\left.I \otimes_{\mathbb{C}} O b_{F_{\alpha}}\right|_{x}
$$

gives us an infinitesimal lifting problem

$$
\bar{g}^{\prime}: \operatorname{Spec} \bar{B} \longrightarrow F_{\alpha} \stackrel{\imath}{\longrightarrow} X_{\alpha}
$$

for $X_{\alpha}$ with obstruction class

$$
\left.o b_{X_{\alpha}}\left(\phi_{\alpha}, \bar{g}^{\prime}, B, \bar{B}\right) \in I \otimes_{\mathbb{C}} O b_{X_{\alpha}}\right|_{x}
$$

where $x \in F$ is the image of the closed point in $\operatorname{Spec} \bar{B}$ by $\bar{g}$. Since $\bar{g}$ is $T$-invariant,

$$
o b_{X_{\alpha}}\left(\phi_{\alpha}, \bar{g}^{\prime}, B, \bar{B}\right)=\left.\left.o b_{F_{\alpha}}\left(\phi_{\alpha}^{F}, \bar{g}, B, \bar{B}\right) \in I \otimes_{\mathbb{C}} O b_{F_{\alpha}}\right|_{x} \subset I \otimes_{\mathbb{C}} O b_{X_{\alpha}}\right|_{x} .
$$

Since the perfect obstruction theories $\left.E_{\alpha}\right|_{X_{\alpha \beta}}$ and $\left.E_{\beta}\right|_{X_{\alpha \beta}}$ give the same obstruction assignment for $X_{\alpha \beta}=X_{\alpha} \times_{X} X_{\beta}$, we find that the same holds for $\left.E_{\alpha}\right|_{F_{\alpha \beta}} ^{f i x}$ and $\left.E_{\beta}\right|_{F_{\alpha \beta}} ^{f i x}$. This proves the lemma.

Recall that in 17, the authors proved the torus localization formula assuming the existence of

(1) a global $T$-equivariant embedding of $X$ into a smooth Deligne-Mumford stack and

(2) a global resolution $\left[E^{-1} \rightarrow E^{0}\right]$ of the perfect obstruction theory $E \rightarrow \mathbb{L}_{X}$ by locally free sheaves $E^{-1}$ and $E^{0}$.

In [6. §3], (1) was completely removed and (2) was replaced by a weaker assumption that the virtual normal bundle $N^{\text {vir }}=\left(\left.E\right|_{F} ^{m v}\right)^{\vee}$ admits a global 2-term resolution $\left[N_{0} \rightarrow N_{1}\right]$ by locally free sheaves $N_{0}, N_{1}$ over $F$.

For the torus localization formula in the setting of semi-perfect obstruction theory, we assume the following.

Assumption 4.3. There is a homomorphism

$$
N^{\mathrm{vir}}=\left[N_{0} \rightarrow N_{1}\right]
$$

of locally free sheaves on $F$ whose cokernel $h^{1}\left(N^{\mathrm{vir}}\right)$ is isomorphic to $\left.O b_{X}\right|_{F} ^{m v}$ such that there is an isomorphism

$$
\left.N^{\mathrm{vir}}\right|_{F_{\alpha}} \cong N_{\alpha}^{\mathrm{vir}}=\left(\left.E_{\alpha}\right|_{F_{\alpha}} ^{m v}\right)^{\vee}, \quad \forall \alpha
$$

in the derived category $D\left(\left[F_{\alpha} / T\right]\right)$ whose $h^{1}$ is the restriction of the isomorphism $\left.h^{1}\left(N^{\text {vir }}\right) \cong O b_{X}\right|_{F} ^{m v}$ to $F_{\alpha}$.

Definition 4.4. Under Assumption 4.3, the Euler class of $N^{\text {vir }}$ is

$$
e\left(N^{\mathrm{vir}}\right)=\frac{e\left(N_{0}\right)}{e\left(N_{1}\right)} \in A_{*}(F) \otimes_{\mathbb{Q}} \mathbb{Q}\left[t, t^{-1}\right]
$$

where $e(-)$ denotes the equivariant top Chern class and $t$ is the generator of the equivariant Chow ring $A_{T}^{*}(p t)$. 
Theorem 4.5. Under Assumption 4.3, we have

$$
[X]^{\mathrm{vir}}=\imath_{*} \frac{[F]^{\mathrm{vir}}}{e\left(N^{\mathrm{vir}}\right)} \in A_{*}^{T}(X) \otimes_{\mathbb{Q}[t]} \mathbb{Q}\left[t, t^{-1}\right]
$$

where $[X]^{\text {vir }}$ and $[F]^{\text {vir }}$ are the virtual fundamental classes of $X$ and $F$ with respect to the semi-perfect obstruction theories $\left\{\phi_{\alpha}: E_{\alpha} \rightarrow \mathbb{L}_{X_{\alpha}}\right\}$ and $\left\{\phi_{\alpha}^{F}\right.$ : $\left.E_{\alpha}^{F} \rightarrow \mathbb{L}_{F_{\alpha}}\right\}$ using the notation above.

To prove Theorem 4.5, we introduce another semi-perfect obstruction theory for $F$, whose obstruction sheaf is enlarged by $N_{1}$ so that the inclusion $\imath: F_{\alpha} \rightarrow X_{\alpha}$ is virtually smooth. Let

$$
\widetilde{E}_{\alpha}^{F}=\left.E_{\alpha}^{F} \oplus N_{1}\right|_{F_{\alpha}} ^{\vee}[1] \text { and } \tilde{\phi}_{\alpha}^{F}: \widetilde{E}_{\alpha}^{F} \longrightarrow E_{\alpha}^{F} \stackrel{\phi_{\alpha}^{F}}{\longrightarrow} \mathbb{L}_{F_{\alpha}}
$$

where the first arrow $\widetilde{E}_{\alpha}^{F} \rightarrow E_{\alpha}^{F}$ is the projection to the direct summand $E_{\alpha}^{F}$ defined in (4.4). Since $\phi_{\alpha}^{F}: E_{\alpha}^{F} \rightarrow \mathbb{L}_{F_{\alpha}}$ is a perfect obstruction theory by Lemma 4.2, $\tilde{\phi}_{\alpha}^{F}$ is also a perfect obstruction theory for $F_{\alpha}$. Since the obstruction sheaves $\left\{h^{1}\left(\left(E_{\alpha}^{F}\right)^{\vee}\right)\right\}$ glue to $O b_{F}=\left.O b_{X}\right|_{F} ^{f i x}$, the obstruction sheaves

$$
h^{1}\left(\left(\widetilde{E}_{\alpha}^{F}\right)^{\vee}\right)=h^{1}\left(\left(E_{\alpha}^{F}\right)^{\vee}\right) \oplus h^{1}\left(\left.N_{1}\right|_{F_{\alpha}}[-1]\right)
$$

glue to

$$
\widetilde{O b}_{F}=O b_{F} \oplus N_{1} \text {. }
$$

As $\tilde{\phi}_{\alpha}^{F}$ factors through $\phi_{\alpha}^{F}$ in (4.10), $\left.\tilde{\phi}_{\alpha}^{F}\right|_{F_{\alpha \beta}}$ and $\left.\tilde{\phi}_{\beta}^{F}\right|_{F_{\alpha \beta}}$ give the same obstruction assignment where $F_{\alpha \beta}=F_{\alpha} \times_{F} F_{\beta}=X_{\alpha \beta} \times_{X} F$. Hence we proved the following.

Lemma 4.6. (4.10) defines a semi-perfect obstruction theory on F with obstruction sheaf $\widetilde{O b}_{F}=O b_{F} \oplus N_{1}$ where $O b_{F}=\left.O b_{X}\right|_{F} ^{f i x}$ is the obstruction sheaf of $F$ with respect to the semi-perfect obstruction theory in Lemma 4.2.

Note that the new semi-perfect obstruction theory $\left\{\left(\widetilde{E}_{\alpha}^{F}, \tilde{\phi}_{\alpha}^{F}\right)\right\}$ fits into the commutative diagram

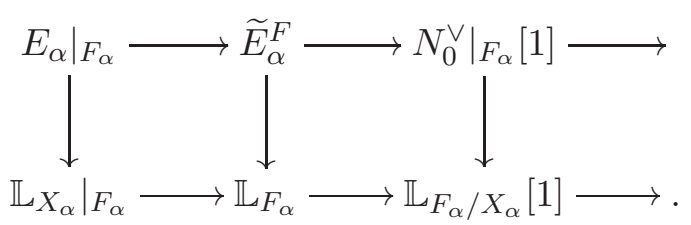

Since $F \hookrightarrow X$ is an embedding, the normal cone $\mathbf{c}_{F / X}=C_{F / X}$ embeds into the normal sheaf $\mathfrak{N}_{F / X}$ which is the kernel of (4.7). Hence we have the embedding

$$
\mathbf{c}_{F / X} \hookrightarrow N_{0} .
$$

This gives us the pullback map

$$
\imath^{!}: A_{*}(X) \longrightarrow A_{*}(F)
$$


defined by

$$
\imath^{!}[B]=0 ! N_{0}\left[\mathbf{c}_{B \times{ }_{X} F / B}\right]
$$

for any prime cycle $B$ in $X$ via the embedding

$$
\mathbf{c}_{B \times{ }_{X} F / B} \subset \mathbf{c}_{F / X} \subset N_{0} .
$$

Proposition 4.7. $\imath^{!}[X]^{\mathrm{vir}}=e\left(N_{1}\right) \cap[F]^{\mathrm{vir}}$.

Proof. Since $[X]^{\mathrm{vir}}=0 !_{\mathrm{Ob}}{ }_{\mathrm{C}}\left[\mathcal{C}_{X}\right]$ by definition, we have $\imath^{!}[X]^{\mathrm{vir}}=\imath^{!} 0_{\mathrm{Ob}}{ }_{b_{X}}\left[\mathcal{C}_{X}\right]$. Recall that $\mathcal{C}_{X}$ is the substack of $O b_{X}$ whose pullback

$$
\left.\mathcal{C}_{X}\right|_{X_{\alpha}} \in A_{*}\left(\left.O b_{X}\right|_{X_{\alpha}}\right)=A_{*}\left(h^{1}\left(E_{\alpha}^{\vee}\right)\right)
$$

to $X_{\alpha}$ is the image of the intrinsic normal cone $\mathbf{c}_{X_{\alpha}} \in A_{*}\left(h^{1} / h^{0}\left(E_{\alpha}^{\vee}\right)\right)$. Since $F \subset X \subset \mathcal{C}_{X}$, the normal cone $\mathbf{c}_{F / \mathcal{C}_{X}} \in A_{*}\left(\left.O b_{X}\right|_{F} \oplus N_{0}\right)$ is defined as a substack whose restriction $\left.\mathbf{c}_{F / \mathcal{C}_{X}}\right|_{F_{\alpha}} \in A_{*}\left(\left.\left.O b_{X}\right|_{F_{\alpha}} \oplus N_{0}\right|_{F_{\alpha}}\right)$ to $F_{\alpha}=F \times_{X} X_{\alpha}$

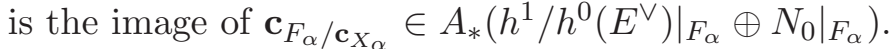

Our proof of Proposition 4.7 will follow from the two lemmas below.

Lemma 4.8. $\imath^{!}[X]^{\mathrm{vir}}=0 !_{\left.O b_{X}\right|_{F} \oplus N_{0}}\left[\mathbf{c}_{F / \mathcal{C}_{X}}\right]$.

Proof. Let $\mathcal{C}_{X}=\sum n_{i} B_{i}$ where $B_{i}$ are distinct prime cycles in $O b_{X}$. Let $\left(f_{i}: S_{i} \rightarrow X, \mathcal{V}_{i} \rightarrow f_{i}^{*} O b_{X}, \tilde{B}_{i}\right)$ be a proper representative of $B_{i}$ and let $F_{S_{i}}=F \times_{X} S_{i}$ so that we have the commutative diagram

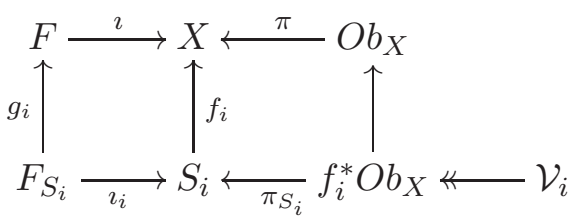

Let $0_{\mathcal{V}_{i}}^{!}\left[\tilde{B}_{i}\right]=\tilde{T}_{i} \in A_{*}\left(S_{i}\right)$ and $T_{i}=\frac{1}{\operatorname{deg} f_{i}} f_{i_{*}} \tilde{T}_{i} \in A_{*}(X)$. Let $\tilde{\pi}_{i}: \mathcal{V}_{i} \rightarrow S_{i}$ denote the bundle projection. Then $\left[\tilde{B}_{i}\right]=\tilde{\pi}_{i}^{*}\left[\tilde{T}_{i}\right] \in A_{*}\left(\mathcal{V}_{i}\right)$ and

$$
[X]^{\text {vir }}=0 !_{O b_{X}}\left[\mathcal{C}_{X}\right]=\sum_{i} \frac{n_{i}}{\operatorname{deg} f_{i}} f_{i_{*}} 0 \dot{\mathcal{V}}_{i}\left[\tilde{B}_{i}\right]=\sum_{i} n_{i} T_{i}
$$

Hence the left side of Lemma 4.8 is

$$
\imath^{!}[X]^{\mathrm{vir}}=\imath^{!} \sum_{i} n_{i} T_{i}=\sum_{i} n_{i} 0_{N_{0}}\left[\mathbf{c}_{F \times X} T_{i} / T_{i}\right] .
$$

By the proof of [14, Proposition 1.9], the rational equivalence $\tilde{B}_{i} \sim \tilde{\pi}_{i}^{*}\left[\tilde{T}_{i}\right]$ is given by admissible rational functions and hence we have $\left[B_{i}\right]=\left[\pi^{-1}\left(T_{i}\right)\right] \in$ $A_{*}\left(O b_{X}\right)$ where $\pi: O b_{X} \rightarrow X$ is the projection. Since $F \subset X$, we have

$$
\mathbf{c}_{F \times X_{X} \pi^{-1}\left(T_{i}\right) / \pi^{-1}\left(T_{i}\right)}=\mathbf{c}_{F \times_{X} T_{i} / T_{i}} \times\left._{F} O b_{X}\right|_{F}
$$

and the right side of Lemma 4.8 is

$$
0 !{ }_{\left.N_{0} \oplus O b_{X}\right|_{F}}\left[\mathbf{c}_{F / \mathcal{C}_{X}}\right]=\sum_{i} n_{i} 0_{\left.N_{0} \oplus O b_{X}\right|_{F}}\left[\mathbf{c}_{F \times_{X} B_{i} / B_{i}}\right]
$$




$$
=\sum_{i} n_{i} 00_{\left.N_{0} \oplus O b_{X}\right|_{F}}\left[\mathbf{c}_{F \times_{X} T_{i} / T_{i}} \times\left._{F} O b_{X}\right|_{F}\right]=\sum_{i} n_{i} 0_{N_{0}}\left[\mathbf{c}_{F \times{ }_{X} T_{i} / T_{i}}\right] .
$$

The lemma follows from (4.15) and (4.16).

Lemma 4.9. There exist a coherent sheaf $\Xi$ on $F \times \mathbb{P}^{1}$ such that

$$
\left.\Xi\right|_{F \times\{s\}}=\left\{\begin{array}{cc}
\widetilde{O b}_{F}=O b_{F} \oplus N_{1} & s \neq 0 \\
\left.O b_{X}\right|_{F} \oplus N_{0} & s=0 .
\end{array}\right.
$$

and a rational equivalence of cycles

$$
\left[\mathbf{c}_{F / \mathcal{C}_{X}}\right] \sim\left[\mathcal{C}_{F}\right] \in A_{*}(\Xi)
$$

Proof. Consider the double deformation spaces

$$
M_{\alpha}=M_{F_{\alpha} \times \mathbb{P}^{1} / M_{X_{\alpha}}^{\circ}}^{\circ} \longrightarrow \mathbb{P}^{1} \times \mathbb{P}^{1}
$$

from [26] such that

$$
\left.M_{\alpha}\right|_{\{t\} \times \mathbb{P}^{1}}=\left\{\begin{array}{cc}
M_{X_{\alpha}}^{\circ} & t \neq 0 \\
\mathbf{c}_{F_{\alpha} \times \mathbb{P}^{1} / M_{X_{\alpha}}^{\circ}} & t=0
\end{array}\right.
$$

where $M_{X_{\alpha}}^{\circ} \rightarrow \mathbb{P}^{1}$ is the deformation space such that

$$
\left.M_{X_{\alpha}}^{\circ}\right|_{s}=\left\{\begin{array}{cc}
\mathrm{pt} & s \neq 0 \\
\mathbf{c}_{X_{\alpha}} & s=0
\end{array}\right.
$$

Here $\mathbf{c}_{X_{\alpha}}$ is the intrinsic normal cone of $X_{\alpha}$. Note that

$$
\left.\mathbf{c}_{F_{\alpha} \times \mathbb{P}^{1} / M_{X_{\alpha}}^{\circ}}\right|_{s}=\left\{\begin{array}{cc}
\mathbf{c}_{F_{\alpha} / \mathbf{c}_{X_{\alpha}}} & s=0 \\
\mathbf{c}_{F_{\alpha}} & s \neq 0 .
\end{array}\right.
$$

From (4.1), (4.4) and (4.10), we have a morphism

$$
\lambda:\left.E_{\alpha}\right|_{F_{\alpha}} \longrightarrow \widetilde{E}_{\alpha}^{F}
$$

whose cone is the locally free sheaf $\left.N_{0}\right|_{F_{\alpha}} ^{\vee}$. Let $\xi_{\alpha}$ be the cone of the morphism

$$
\left(x_{0} \cdot \mathrm{id}, x_{1} \cdot \lambda\right):\left.\left.p_{1}^{*} E_{\alpha}\right|_{F_{\alpha}} \otimes p_{2}^{*} \mathcal{O}_{\mathbb{P}^{1}}(-1) \longrightarrow p_{1}^{*} E_{\alpha}\right|_{F_{\alpha}} \oplus p_{1}^{*} \widetilde{E}_{\alpha}^{F}
$$

where $x_{0}, x_{1}$ are the homogeneous coordinates of $\mathbb{P}^{1}$ and $p_{1}, p_{2}$ are the projections from $F \times \mathbb{P}^{1}$ to $F$ and $\mathbb{P}^{1}$ respectively.

Since $\mathbf{c}_{F_{\alpha} \times \mathbb{P}^{1} / M_{X_{\alpha}}^{\circ}} \subset h^{1} / h^{0}\left(\xi_{\alpha}^{\vee}\right)$, we have a rational equivalence

$$
\left[\mathbf{c}_{F_{\alpha} / \mathbf{c}_{X_{\alpha}}}\right] \sim\left[\mathbf{c}_{F_{\alpha}}\right]
$$

in $A_{*}\left(h^{1} / h^{0}\left(\xi_{\alpha}^{\vee}\right)\right)$ and hence an equivalence

$$
\left[\mathbf{c}_{F_{\alpha} / \mathcal{C}_{X_{\alpha}}}\right] \sim\left[\mathcal{C}_{F_{\alpha}}\right]
$$

in $A_{*}\left(h^{1}\left(\xi_{\alpha}^{\vee}\right)\right)$ where $\left[\mathcal{C}_{F_{\alpha}}\right]$ is the image of $\left[\mathbf{c}_{F_{\alpha}}\right]$ by the canonical morphism $h^{1} / h^{0}\left(\xi_{\alpha}^{\vee}\right) \rightarrow h^{1}\left(\xi_{\alpha}^{\vee}\right)$.

Note that $\left\{\mathcal{C}_{F_{\alpha}}\right\},\left\{\mathcal{C}_{X_{\alpha}}\right\}$ and $\left\{\mathbf{c}_{F_{\alpha} / \mathcal{C}_{X_{\alpha}}}\right\}$ all glue to

$$
\left[\mathcal{C}_{F}\right] \in A_{*}\left(O b_{F}\right), \quad\left[\mathcal{C}_{X}\right] \in A_{*}\left(O b_{X}\right), \quad \text { and } \quad\left[\mathbf{c}_{F / \mathcal{C}_{X}}\right]
$$


respectively. The canonical equivalences (4.20) glue to an equivalence

$$
\left[\mathbf{c}_{F / \mathcal{C}_{X}}\right] \sim\left[\mathcal{C}_{F}\right] \in A_{*}(\Xi) .
$$

To prove (4.17), we take a more careful look at (4.19). To simplify the notation, we will often $\operatorname{drop} p_{1}^{*}, p_{2}^{*},\left.\cdot\right|_{F_{\alpha}}$ when the meaning is clear from the context. For example, $\left.E_{\alpha}\right|_{F_{\alpha}}(-1)$ means $\left.p_{1}^{*} E_{\alpha}\right|_{F_{\alpha}} \otimes p_{2}^{*} \mathcal{O}_{\mathbb{P}^{1}}(-1)$ and $N_{1}^{\vee}$ often means $\left.N_{1}\right|_{F_{\alpha}} ^{\vee}$. By (4.1), (4.4), (4.10) and (4.8), we have

$$
\begin{gathered}
\left.p_{1}^{*} E_{\alpha}\right|_{F_{\alpha}} \otimes p_{2}^{*} \mathcal{O}_{\mathbb{P}^{1}}(-1)=\left[N_{1}^{\vee} \rightarrow N_{0}^{\vee}\right](-1) \oplus E_{\alpha}^{F}(-1), \\
\left.p_{1}^{*} E_{\alpha}\right|_{F_{\alpha}} \oplus p_{1}^{*} \widetilde{E}_{\alpha}^{F}=\left[N_{1}^{\vee} \rightarrow N_{0}^{\vee}\right] \oplus\left[N_{1}^{\vee} \rightarrow 0\right] \oplus E_{\alpha}^{F} \oplus E_{\alpha}^{F} .
\end{gathered}
$$

The cone $\xi_{\alpha}^{f i x}$ of the fixed part

$$
E_{\alpha}^{F}(-1) \stackrel{\left(x_{0}, x_{1}\right)}{\longrightarrow} E_{\alpha}^{F} \oplus E_{\alpha}^{F}
$$

is certainly $E_{\alpha}^{F}(1)$ and $\left\{h^{1}\left(E_{\alpha}^{F}(1)^{\vee}\right)\right\}$ glue to the sheaf $p_{1}^{*} O b_{F} \otimes p_{2}^{*} \mathcal{O}_{\mathbb{P}^{1}}(1)$ on $F \times \mathbb{P}^{1}$. By direct computation, the cone $\xi_{\alpha}^{m v}$ of the moving part has

$$
h^{1}\left(\left(\xi_{\alpha}^{m v}\right)^{\vee}\right)=h^{1}\left(N_{0} \stackrel{\left(d, 0,-x_{0}\right)}{\longrightarrow} N_{1} \oplus N_{1} \oplus N_{0}(1) \stackrel{\left(-x_{0},-x_{1},-d\right)}{\longrightarrow} N_{1}(1)\right)
$$

where $d$ is the differential of $N^{\text {vir }}=\left[N_{0} \stackrel{d}{\longrightarrow} N_{1}\right]$. Since $N_{0}, N_{1}, d, x_{0}, x_{1}$ are globally defined on $F \times \mathbb{P}^{1}$, we find that $\left\{h^{1}\left(\xi_{\alpha}\right)\right\}$ glue to a sheaf $\Xi$ on $F \times \mathbb{P}^{1}$. Finally (4.17) follows from (4.22).

Now we can complete our proof of Proposition 4.7. By Lemma 4.9,

$$
0 !\left[\mathcal{C}_{F}\right]=0 !\left[\mathbf{c}_{F / \mathcal{C}_{X}}\right]
$$

Since $\left.\mathcal{C}_{F} \subset \Xi\right|_{F \times\{\infty\}}=O b_{F} \oplus N_{1}$, we have

$$
\left.0 ! \mathcal{C}_{F}\right]=0 !_{O b_{F} \oplus N_{1}}\left[\mathcal{C}_{F}\right]=e\left(N_{1}\right) \cap[F]^{\text {vir }} .
$$

Likewise, since $\left.\mathbf{c}_{F / \mathcal{C}_{X}} \subset \Xi\right|_{F \times\{0\}}=\left.O b_{X}\right|_{F} \oplus N_{0}$, by Lemma 4.8, we have

$$
0 !\left[\mathbf{c}_{F / \mathcal{C}_{X}}\right]=\left.0 ! b_{X}\right|_{F} \oplus N_{0}\left[\mathbf{c}_{F / \mathcal{C}_{X}}\right]=l^{!}[X]^{\mathrm{vir}} .
$$

The proposition follows from (4.23), (4.24) and (4.25).

Now we can prove Theorem 4.5 .

Proof of Theorem 4.5. By [28, Theorem 6.3.5], the inclusion $\imath: F \rightarrow X$ induces an isomorphism

$$
\imath_{*}: A_{*}^{T}(F) \otimes_{\mathbb{Q}[t]} \mathbb{Q}\left[t, t^{-1}\right] \longrightarrow A_{*}^{T}(X) \otimes_{\mathbb{Q}[t]} \mathbb{Q}\left[t, t^{-1}\right] .
$$

Hence we have a class $\gamma \in A_{*}^{T}(F) \otimes_{\mathbb{Q}[t]} \mathbb{Q}\left[t, t^{-1}\right]$ such that $\imath_{*} \gamma=[X]^{\text {vir }}$.

By (4.14),,$![B]=0 !{ }_{N_{0}}\left(\mathbf{c}_{B \times{ }_{X} F / B}\right)=0 !{ }_{N_{0}}[B]=e\left(N_{0}\right) \cap[B]$ if $B \subset F$. Hence we have

$$
\imath^{!}[X]^{\mathrm{vir}}=\imath^{!} \imath_{*} \gamma=e\left(N_{0}\right) \cap \gamma .
$$

By Proposition 4.7, we obtain

$$
\gamma \cap e\left(N_{0}\right)=e\left(N_{1}\right) \cap[F]^{\text {vir }} .
$$


Since $e\left(N^{\text {vir }}\right)=e\left(N_{0}\right) / e\left(N_{1}\right)$,

$$
[X]^{\text {vir }}=\imath_{*} \gamma=\imath_{*} \frac{[F]^{\text {vir }}}{e\left(N^{\text {vir }}\right)}
$$

as desired.

\section{TORus localization OF COSECTION LOCALIZED VIRTUAL CYCLES FOR SEMI-PERFECT OBSTRUCTION THEORY}

In this section, we generalize the torus localization formula of cosection localized virtual cycle (cf. [6, §3]) to the setting of semi-perfect obstruction theory.

Let $X$ be a Deligne-Mumford stack equipped with

(1) an action of $T=\mathbb{C}^{*}$,

(2) a semi-perfect obstruction theory

$$
\left(X_{\alpha} \rightarrow X, \phi_{\alpha}: E_{\alpha} \rightarrow \mathbb{L}_{X_{\alpha}}\right), \quad \text { and }
$$

(3) a $T$-invariant cosection

$$
\sigma: O b_{X} \longrightarrow \mathcal{O}_{X}
$$

of the obstruction sheaf $O b_{X}$ of the semi-perfect obstruction theory.

Let $X(\sigma)$ denote the zero locus of $\sigma$, that is the closed substack defined by the image of $\sigma$. By Lemma 4.2, we have an induced semi-perfect obstruction theory

$$
\left(F_{\alpha} \rightarrow F, \phi_{\alpha}^{F}: E_{\alpha}^{F} \rightarrow \mathbb{L}_{F_{\alpha}}\right)
$$

on $F$. Since $\sigma$ is $T$-invariant, the restriction $\left.\sigma\right|_{F}$ of $\sigma$ to $F$ factors through a homomorphism

$$
\sigma_{F}: O b_{F} \longrightarrow \mathcal{O}_{F}
$$

whose zero locus is $F(\sigma)=F \times_{X} X(\sigma)$.

By Theorem 3.1, we have cosection localized virtual fundamental classes

$$
[X]_{\mathrm{loc}}^{\mathrm{vir}} \in A_{*}(X(\sigma)), \quad[F]_{\mathrm{loc}}^{\mathrm{vir}} \in A_{*}(F(\sigma)) .
$$

The goal of this section is to prove the following.

Theorem 5.1. Let $\imath: F(\sigma) \rightarrow X(\sigma)$ denote the inclusion. Suppose Assumption 4.3 holds. Then we have

$$
[X]_{\mathrm{loc}}^{\mathrm{vir}}=\imath_{*} \frac{[F]_{\text {loc }}^{\mathrm{vir}}}{e\left(N^{\mathrm{vir}}\right)} \in A_{*}^{T}(X(\sigma)) \otimes_{\mathbb{Q}[t]} \mathbb{Q}\left[t, t^{-1}\right] .
$$

As in 4 , Theorem 5.1 follows from the following lemma.

Lemma 5.2. $\imath^{!}[X]_{\text {loc }}^{\mathrm{vir}}=e\left(N_{1}\right) \cap[F]_{\text {loc }}^{\mathrm{vir}}$. 
Proof. The proof is identical to that of Proposition 4.7. once we show that the rational equivalence in Lemma 4.9 lies in

$$
\Xi(\sigma)=\left.\Xi\right|_{F(\sigma) \times \mathbb{P}^{1}} \cup \operatorname{ker}\{\sigma: \Xi \rightarrow \mathcal{O}\} .
$$

Its proof is identical to that of [6, Lemma 2.5].

Proof of Theorem 5.1. The proof of Theorem 4.5 also proves Theorem 5.1 if we replace $F, X,[F]^{\text {vir }}$ and $[X]^{\text {vir }}$ by $F(\sigma), X(\sigma),[F]_{\text {loc }}^{\text {vir }}$ and $[X]_{\text {loc }}^{\text {vir }}$ respectively.

\section{Dual obstruction CONE AND Virtual Signed Euler CHARACTERISTIC}

In this section, we recall the dual obstruction cone $N$ of a DeligneMumford stack $X$ with a perfect obstruction theory by Jiang-Thomas [20] and prove that it admits a semi-perfect obstruction theory (Theorem 6.2). The cosection localization (Theorem 3.1) and the torus localization (Theorem 4.5) above for semi-perfect obstruction theory now establish the JiangThomas theory of signed Euler characteristic (cf. [20]) without any assumption on the derived geometry of $X$.

6.1. Dual obstruction cone. In this subsection, we recall the dual obstruction cone by Jiang-Thomas [20]. Let $X$ be a Deligne-Mumford stack equipped with a perfect obstruction theory

$$
E \longrightarrow \mathbb{L}_{X}
$$

whose obstruction sheaf is $O b_{X}=h^{1}\left(E^{\vee}\right)$.

Definition 6.1. The dual obstruction cone of $X$ is defined as

$$
N=\operatorname{Spec}_{X}(\operatorname{Sym} \mathcal{F}) \stackrel{\pi}{\longrightarrow} X, \quad \mathcal{F}=O b_{X}
$$

which represents the functor

$$
\text { (Schemes over } X) \longrightarrow \text { (Sets), }(S \stackrel{f}{\longrightarrow} X) \mapsto \operatorname{Hom}_{S}\left(f^{*} \mathcal{F}, \mathcal{O}_{S}\right)
$$

i.e. giving a morphism $S \rightarrow N$ over $X$ amounts to giving a morphism $S \stackrel{f}{\longrightarrow} X$ together with a homomorphism $f^{*} \mathcal{F} \rightarrow \mathcal{O}_{S}$.

Finding a cosection $\sigma: \mathcal{F} \rightarrow \mathcal{O}_{X}$ of $\mathcal{F}$ is the same as finding a section $s: X \rightarrow N$ of (6.2).

By a standard argument on perfect obstruction theory, there is an étale cover $\left\{X_{\alpha} \rightarrow X\right\}$ of $X$ such that $X_{\alpha}$ is the zero locus of a section $s_{\alpha}$ of a trivial vector bundle $V_{\alpha}$ on a smooth variety $A_{\alpha}$

$$
X_{\alpha}=\operatorname{zero}\left(s_{\alpha}\right) \longrightarrow \stackrel{A_{\alpha}}{V_{\alpha}}
$$


and that the perfect obstruction theory $E$ restricted to $X_{\alpha}$ is

$$
\left.E\right|_{X_{\alpha}} \cong\left[\left.\left.V_{\alpha}^{\vee}\right|_{X_{\alpha}} \stackrel{\left(d s_{\alpha}\right)^{\vee}}{\longrightarrow} \Omega_{A_{\alpha}}\right|_{X_{\alpha}}\right]
$$

Since the obstruction sheaf $\left.O b_{X}\right|_{X_{\alpha}}$ of the perfect obstruction theory $\left.E\right|_{X_{\alpha}}$ is $\operatorname{coker}\left(d s_{\alpha}\right), N_{\alpha}:=\left.N\right|_{X_{\alpha}}$ is the subscheme of $\left.V_{\alpha}^{\vee}\right|_{X_{\alpha}}$ defined by the vanishing of the section

$$
\left(d s_{\alpha}\right)^{\vee}:\left.\left.V_{\alpha}^{\vee}\right|_{X_{\alpha}} \longrightarrow \pi_{\alpha}^{*} \Omega_{A_{\alpha}}\right|_{X_{\alpha}}
$$

where $\pi_{\alpha}:\left.V_{\alpha}\right|_{X_{\alpha}} ^{\vee} \rightarrow X_{\alpha}$ is the projection.

If we let $x_{1}, \cdots, x_{n}$ be coordinates for $A_{\alpha}$ and $y_{1}, \cdots, y_{r}$ be coordinates of the fiber of $V_{\alpha}^{\vee} \rightarrow A_{\alpha}, N_{\alpha}=\left.N\right|_{X_{\alpha}}$ is defined by the ideal generated by

$$
\left\{s_{i}\right\}_{1 \leq i \leq n}, \quad\left\{\sum_{i} y_{i} \frac{\partial s_{i}}{\partial x_{j}}\right\}_{1 \leq j \leq r}
$$

where $s_{i}=s_{i}\left(x_{1}, \cdots, x_{n}\right)$ are the coordinate functions of $s_{\alpha}$. Let

$$
\tilde{s}_{\alpha}: V_{\alpha}^{\vee} \stackrel{s_{\alpha}}{\longrightarrow} \mathbb{C}
$$

be the function on the dual bundle $V_{\alpha}^{\vee}$ of $V_{\alpha}$, defined by the section $s_{\alpha}$ of $V_{\alpha}$ over $A_{\alpha}$ :

$$
\tilde{s}_{\alpha}=\sum_{i=1}^{r} y_{i} s_{i} .
$$

Hence the critical locus of $\tilde{s}_{\alpha}$ is defined by the vanishing of

$$
d \tilde{s}_{\alpha}=\sum_{i} s_{i} d y_{i}+\sum_{j}\left(\sum_{i} y_{i} \frac{\partial s_{i}}{\partial x_{j}}\right) d x_{j}
$$

and so $N_{\alpha}$ is the critical locus of the function $\tilde{s}_{\alpha}$ defined on the smooth variety $V_{\alpha}^{\vee}$, i.e.

$$
N_{\alpha}=\left.N\right|_{X_{\alpha}}=\operatorname{Crit}\left(\tilde{s}_{\alpha}\right) .
$$

In particular, $N_{\alpha}$ is equipped with a symmetric perfect obstruction theory

$$
F_{\alpha}:=\left[\left.\left.T_{V_{\alpha}^{\vee}}\right|_{N_{\alpha}} \stackrel{d\left(d \tilde{s}_{\alpha}\right)}{\longrightarrow} \Omega_{V_{\alpha}^{\vee}}\right|_{N_{\alpha}}\right] .
$$

6.2. Semi-perfect obstruction theory of $N$. In this subsection, we prove that the dual obstruction cone $N$ in (6.2) has a symmetric semi-perfect obstruction theory defined by (6.8).

Theorem 6.2. The dual obstruction cone $N$ has a symmetric semi-perfect obstruction theory

$$
\left\{N_{\alpha} \rightarrow N\right\}, \quad\left\{F_{\alpha} \rightarrow \mathbb{L}_{N_{\alpha}}\right\}
$$

where $F_{\alpha}$ is defined by (6.8) and $N_{\alpha}=\left.N\right|_{X_{\alpha}}=N \times_{X} X_{\alpha}$. 
Proof. We use the notation and local description of 6.1. Since (6.8) is symmetric, the obstruction sheaves

$$
O b_{N_{\alpha}}=h^{1}\left(F_{\alpha}^{\vee}\right) \cong \Omega_{N_{\alpha}}=\left.\Omega_{N}\right|_{N_{\alpha}}
$$

glue to the cotangent sheaf $\Omega_{N}$. We have to show that the perfect obstruction theories $F_{\alpha}$ and $F_{\beta}$ on

$$
N_{\alpha \beta}:=N_{\alpha} \times_{N} N_{\beta}=N \times_{X} X_{\alpha \beta}
$$

give the same obstruction assignment (cf. \$2).

Let $x \in N_{\alpha \beta}$ be a closed point and let $B$ be an Artin local ring with maximal ideal $m_{B}$. Let $I$ be an ideal of $B$ satisfying $I \cdot m_{B}=0$. Let $\bar{B}=B / I$. Let $\bar{g}: \operatorname{Spec} \bar{B} \rightarrow N_{\alpha \beta}$ be a morphism that sends the closed point of $\operatorname{Spec} \bar{B}$ to $x$. We then have a diagram

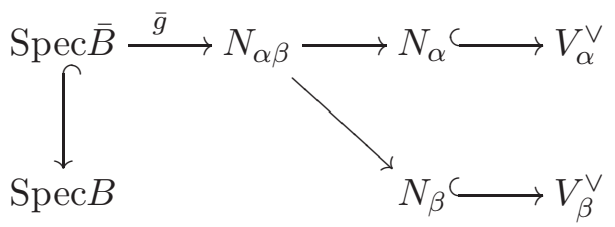

Since $V_{\alpha}^{\vee}$ and $V_{\beta}^{\vee}$ are smooth, there are morphisms

$$
V_{\alpha}^{\vee} \stackrel{g_{\alpha}}{\longleftarrow} \operatorname{Spec} B \stackrel{g_{\beta}}{\longrightarrow} V_{\beta}^{\vee}
$$

that extend (6.12). The functions $\tilde{s}_{\alpha}$ and $\tilde{s}_{\beta}$ in (6.5) give us sections

$$
d \tilde{s}_{\alpha}: V_{\alpha}^{\vee} \longrightarrow \Omega_{V_{\alpha}^{\vee}}, \quad d \tilde{s}_{\beta}: V_{\beta}^{\vee} \longrightarrow \Omega_{V_{\beta}^{\vee}} .
$$

Composing (6.13) and (6.14) with the canonical

$$
\rho_{\alpha}:\left.\left.I \otimes_{\mathbb{C}} \Omega_{V_{\alpha}}\right|_{x} \rightarrow I \otimes_{\mathbb{C}} \Omega_{N_{\alpha}}\right|_{x}, \quad \rho_{\beta}:\left.\left.I \otimes_{\mathbb{C}} \Omega_{V_{\beta}^{\vee}}\right|_{x} \rightarrow I \otimes_{\mathbb{C}} \Omega_{N_{\beta}}\right|_{x}
$$

we obtain the obstruction classes

$$
\begin{gathered}
o b_{\alpha}=\left.\rho_{\alpha}\left(\left.d \tilde{s}_{\alpha} \circ g_{\alpha}\right|_{x}\right) \in I \otimes_{\mathbb{C}} \Omega_{N_{\alpha \beta}}\right|_{x}, \\
o b_{\beta}=\left.\rho_{\beta}\left(\left.d \tilde{s}_{\beta} \circ g_{\beta}\right|_{x}\right) \in I \otimes_{\mathbb{C}} \Omega_{N_{\alpha \beta}}\right|_{x}
\end{gathered}
$$

by [22, Lemma 1.28]. The theorem follows if we show that $o b_{\alpha}=o b_{\beta}$. 
Note that the perfect obstruction theory (6.8) fits into the commutative diagram of exact sequences

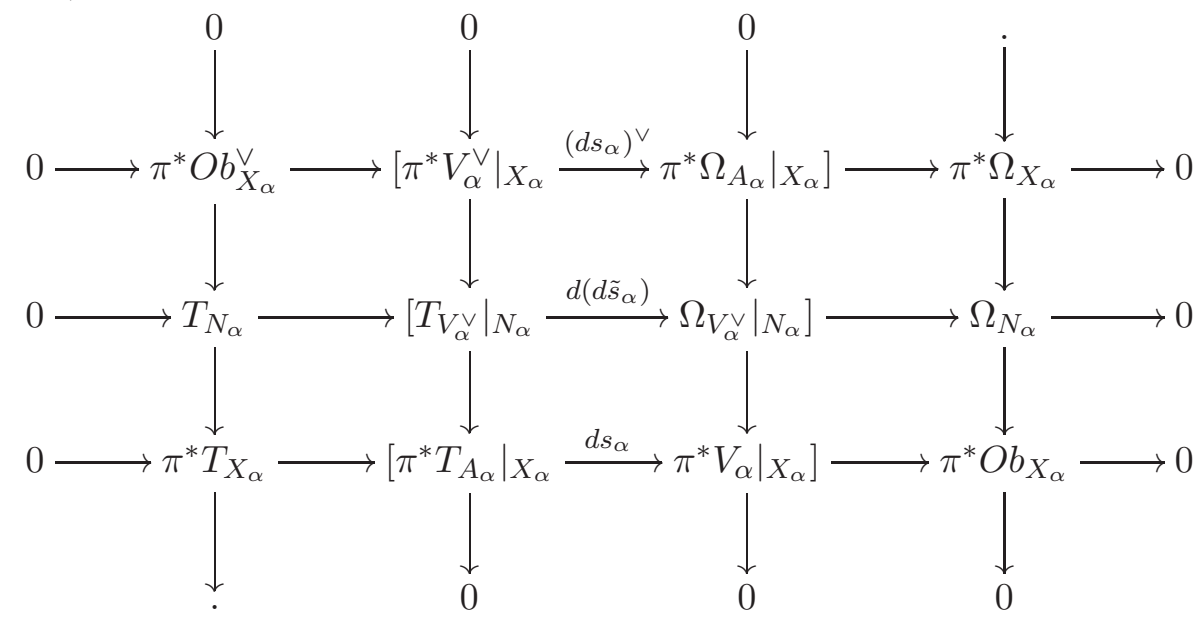

where $\pi: N_{\alpha} \rightarrow X_{\alpha}$ is the projection. Hence we may write

$$
\begin{gathered}
o b_{\alpha}=\hat{o b} \hat{b}_{\alpha}+\overline{o b}_{\alpha} \quad \text { and } \quad b_{\beta}=\hat{o b}_{\beta}+\overline{o b}_{\beta}, \\
\hat{o b_{\alpha}},\left.\hat{o b_{\beta}} \in I \otimes_{\mathbb{C}} \pi^{*} O b_{X_{\alpha \beta}}\right|_{x}, \quad \text { and } \quad \overline{o b}_{\alpha},\left.\overline{o b}_{\beta} \in I \otimes_{\mathbb{C}} \pi^{*} \Omega_{X_{\alpha \beta}}\right|_{x} .
\end{gathered}
$$

where $\hat{o b}$ and $\overline{o b}$ correspond to the first and the second terms respectively of the right side of (6.6). The theorem follows if we show that $\hat{o b}_{\alpha}=\hat{o b} \beta$ and $\overline{o b}_{\alpha}=\overline{o b}{ }_{\beta}$.

We first observe that $\hat{o b}_{\alpha}=\hat{o b}{ }_{\beta}$. This is because they are in fact the obstruction classes for $X_{\alpha}$ and $X_{\beta}$ given by the morphisms

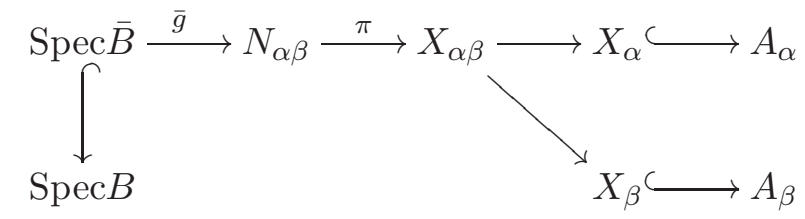

and their extensions

$$
A_{\alpha} \longleftarrow V_{\alpha}^{\vee} \stackrel{g_{\alpha}}{\longleftarrow} \operatorname{Spec} B \stackrel{g_{\beta}}{\longrightarrow} V_{\beta}^{\vee} \longrightarrow A_{\beta} .
$$

These classes coincide because they arise from a perfect obstruction theory (6.1).

Next we show that $\overline{o b}_{\alpha}=\overline{o b}_{\beta}$. Note that the last term of (6.6) is exactly the pullback of

$$
\left(d s_{\alpha}\right)^{\vee}: V_{\alpha}^{\vee} \rightarrow \Omega_{A_{\alpha}} .
$$

The obstruction class $\overline{o b}_{\alpha}$ is the image of the closed point in $\operatorname{Spec} \bar{B}$ by the map

$$
\left.\left.\operatorname{Spec} \bar{B} \stackrel{\bar{g}}{\longrightarrow} N_{\alpha \beta} \hookrightarrow V_{\alpha}^{\vee}\right|_{X_{\alpha \beta}} \stackrel{\left(d s_{\alpha}\right)^{\vee}}{\longrightarrow} \pi^{*} \Omega_{A_{\alpha}}\right|_{X_{\alpha \beta}} \longrightarrow \pi^{*} \Omega_{X_{\alpha \beta}}
$$


and twisting by $I$. By (6.4), we have isomorphisms

$$
\left.\left[\left.\left.V_{\alpha}^{\vee}\right|_{X_{\alpha \beta}} \stackrel{\left(d s_{\alpha}\right)^{\vee}}{\longrightarrow} \Omega_{A_{\alpha}}\right|_{X_{\alpha \beta}}\right] \cong E\right|_{X_{\alpha \beta}} \cong\left[\left.\left.V_{\beta}^{\vee}\right|_{X_{\alpha \beta}} \stackrel{\left(d s_{\beta}\right)^{\vee}}{\longrightarrow} \Omega_{A_{\beta}}\right|_{X_{\alpha \beta}}\right]
$$

that give rise to a commutative diagram

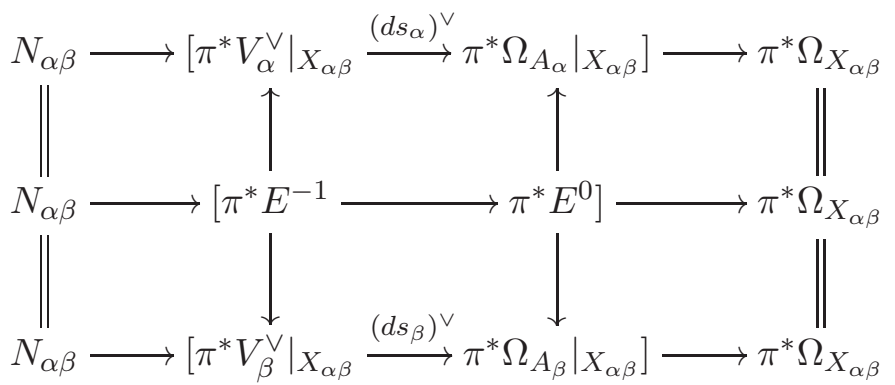

for some locally free sheaves $E^{-1}$ and $E^{0}$ on $X_{\alpha \beta}$. The equality $\overline{o b}_{\alpha}=\overline{o b}_{\beta}$ follows from the commutativity of (6.18). This proves the theorem.

We will see below that the semi-perfect obstruction theory (6.9) of the dual obstruction cone $N$ is $\mathbb{C}^{*}$-equivariant and admits a cosection of the obstruction sheaf $O b_{N}=\Omega_{N}$. So we may apply the torus localization and cosection localization proved in this paper.

Question 6.3. Is the dual obstruction cone $N$ a critical virtual manifold in the sense of [22, Definition 1.5]?

6.3. Localization of the virtual cycle of the dual obstruction cone. Let $X$ be a scheme equipped with a perfect obstruction theory $E$ that gives us the virtual fundamental class $[X]^{\text {vir }}$. We assume that $E$ admits a global resolution $\left[E^{-1} \rightarrow E^{0}\right]$ by locally free sheaves on $X$. Let $\mathcal{F}=O b_{X}=$ $h^{1}\left(E^{\vee}\right)$. The natural grading on $\operatorname{Sym}_{\mathcal{O}_{X}} \mathcal{F}$ determines a $T=\mathbb{C}^{*}$-action on $N=\operatorname{Spec}_{X}(\operatorname{Sym} \mathcal{F})$ whose fixed locus is precisely $X$.

The action of $T$ on $N$ moreover gives us the Euler vector field $v$ on $N$ whose dual is a cosection

$$
\sigma: \Omega_{N} \rightarrow \mathcal{O}_{N}
$$

which is surjective on $N-X$ by [20, $\S 3]$.

By construction, the semi-perfect obstruction theoy of $N$ in Theorem 6.2 is $T$-equivariant and the obstruction sheaf is $O b_{N}=\Omega_{N}$. By (6.17), the virtual normal bundle of the $T$-fixed locus $X$ in $N$ is the dual $E^{\vee}$ of $E$ which admits a global resolution by assumption. Therefore, we can apply the localization theorems (Theorems 3.1 and 4.5) that we proved for semiperfect obstruction theory.

Jiang and Thomas in [20] considered five natural ways to define the notion of virtual signed Euler characteristic of $X$ as follows:

(1) (Ciocan-Fontanine-Kapranov/Fantechi-Göttsche)

$$
e_{1}(X)=\int_{[X]^{\mathrm{vir}}} c_{\mathrm{rk} E}\left(E^{\vee}\right)
$$


(2) (Graber-Pandharipande)

$$
e_{2}(X)=\int_{[X]^{\mathrm{vir}}} \frac{c_{t o p}^{T}\left(E^{0} \otimes \mathbf{t}\right)}{c_{t o p}^{T}\left(E^{-1} \otimes \mathbf{t}\right)},
$$

(3) (Behrend)

$$
e_{3}(X)=e\left(N, \nu_{N}\right)=e\left(X,\left.\nu_{N}\right|_{X}\right)
$$

where $\nu_{N}$ is the Behrend function [1],

(4) (Kiem-Li)

$$
e_{4}(X)=\int_{[N]_{\text {loc }}^{\text {vir }}} 1
$$

for the cosection localized virtual cycle $[N]_{\text {loc }}^{\text {vir }} \in A_{0}(X)$,

(5) $e_{5}(X)=(-1)^{\mathrm{rk} E} e(X)$.

By Theorems 6.2, 3.1 and 4.5, we do not need to assume that $X$ comes from a quasi-smooth derived scheme as in [20]. So the following theorem of Jiang-Thomas holds without any assumption from derived geometry.

Theorem 6.4. 20] Let $X$ be a scheme equipped with a perfect obstruction theory $E$ which admits a global resolution $\left[E^{-1} \rightarrow E^{0}\right]$ by locally free sheaves. Then the five definitions of virtual signed Euler characteristic are well defined and satisfy

$$
e_{1}(X)=e_{2}(X), \quad e_{3}(X)=e_{4}(X)=e_{5}(X) .
$$

\section{REFERENCES}

1. K. Behrend. Donaldson-Thomas type invariants via microlocal geometry. Ann. of Math. (2) 170 (2009), no. 3, 1307-1338.

2. K. Behrend and B. Fantechi. The intrinsic normal cone. Invent. Math. 128 (1997), no. $1,45-88$.

3. V. Bussi. Generalized Donaldson-Thomas theory over fields $K$ neq C. Preprint, arXiv:1403.2403.

4. Y. Cao and N. Leung. Donaldson-Thomas theory for Calabi-Yau 4-folds. Preprint, arXiv:1407.7659.

5. H.-L. Chang and Y.-H. Kiem. Poincaré invariants are Seiberg-Witten invariants. Geom. Topol. 17 (2013), no. 2, 1149-1163.

6. H.-L. Chang, Y.-H. Kiem and J. Li. Torus localization and wall crossing for cosection localized virtual cycles. Preprint, arXiv:1502.00078.

7. H.-L. Chang and J. Li Semi-perfect obstruction theory and Donaldson-Thomas invariants of derived objects. Comm. Anal. Geom. 19 (2011), no. 4, 807830.

8. H.-L. Chang and J. Li. Gromov-Witten invariants of stable maps with fields. Int. Math. Res. Not. IMRN 2012, no. 18, 4163-4217.

9. H.-L. Chang, J. Li and W.-P. Li. Witten's top Chern class via cosection localization. Invent. Math. 200 (2015), no. 3, 10151063

10. H.-L. Chang, J. Li, W.-P. Li and M. C.-C. Liu. Mixed-Spin-P fields of Fermat quintic polynomials. Preprint, arXiv:1505.07532

11. E. Clader. Landau-Ginzburg/Calabi-Yau correspondence for the complete intersections $X_{3,3}$ and $X_{2,2,2,2}$. Preprint, arXiv:1301.5530

12. D. Edidin and W. Graham. Equivariant intersection theory. Invent. Math. 131 (1998), no. 3, 595-634. 
13. H. Fan, T. Jarvis and Y. Ruan. The Witten equation, mirror symmetry, and quantum singularity theory. Ann. of Math. (2) 178 (2013), no. 1, 1106.

14. W. Fulton. Intersection theory. Ergebnisse der Mathematik und ihrer Grenzgebiete. 3. Folge. 2. Springer-Verlag, Berlin, 1998.

15. A. Gholampour and A. Sheshmani. Donaldson-Thomas Invariants of 2-Dimensional sheaves inside threefolds and modular forms. Preprint, arXiv:1309.0050.

16. A. Gholampour and A. Sheshmani. Invariants of pure 2-dimensional sheaves inside threefolds and modular forms. Preprint, arXiv:1305.1334.

17. T. Graber and R. Pandharipande. Localization of virtual cycles. Invent. Math. 135 (1999), no. 2, 487-518.

18. R. Hartshorne. Algebraic Geometry. GTM 52, Springer-Verlag.

19. J. Hu, W.-P. Li, Z. Qin. The Gromov-Witten invariants of the Hilbert schemes of points on surfaces with $p_{g}>0$. Preprint, arXiv:1406.2472

20. Y. Jiang and R. Thomas. Virtual signed Euler characteristics. Preprint, arXiv:1408.2541.

21. Y.-H. Kiem and J. Li. Localizing virtual cycles by cosections. J. Amer. Math. Soc. 26 (2013), no. 4, 1025-1050.

22. Y.-H. Kiem and J. Li. Categorification of Donaldson-Thomas invariant via perverse sheaves. Preprint, arXiv: 1212.6444.

23. Y.-H. Kiem and J. Li. Low degree $G W$ invariants of spin surfaces. Pure Appl. Math. Q. 7 (2011), no. 4, Special Issue: In memory of Eckart Viehweg, 1449-1475.

24. Y.-H. Kiem and J. Li. Low degree $G W$ invariants of surfaces II. Sci. China Math. 54 (2011), no. 8, 1679-1706.

25. Y.-H. Kiem and J. Li. A wall crossing formula of Donaldson-Thomas invariants without Chern-Simons functional. Asian J. Math. 17 (2013), no. 1, 63-94.

26. B. Kim, A. Kresch and T. Pantev. Functoriality in intersection theory and a conjecture of Cox, Katz, and Lee. J. Pure Appl. Algebra 179 (2003), no. 1-2, 127-136.

27. M. Kool and R. Thomas. Reduced classes and curve counting on surfaces I: theory. Preprint, arXiv:1112.3069

28. A. Kresch. Cycle groups for Artin stacks. Invent. Math. 138 (1999), no. 3, 495-536.

29. G. Laumon and L. Moret-Bailly. Champs algebriques, Ergebnisse der Mathematik 39, Springer-Verlag, Berlin (2000).

30. J. Li . A degeneration formula of $G W$-invariants. J. Differential Geom. 60 (2002), no. 2, 199-293.

31. J. Li and G. Tian. Virtual moduli cycles and Gromov-Witten invariants of algebraic varieties. J. Amer. Math. Soc. 11 (1998), no. 1, 119-174.

32. W.-P. Li and Z. Qin. The cohomological crepant resolution conjecture for the HilbertChow morphisms. Preprint, arXiv:1201.3094

33. C. Manolache. Virtual pullbacks. J. Algebraic Geom. 21 (2012), no. 2, 201-245.

34. D. Maulik, R. Pandharipande and R. Thomas. Curves on K3 surfaces and modular forms. With an appendix by A. Pixton. J. Topol. 3 (2010), no. 4, 937-996.

35. R. Pandharipande and A. Pixton. Descendent theory for stable pairs on toric 3-folds. J. Math. Soc. Japan65 (2013), no. 4, 1337-1372.

36. R. Pandharipande and R Thomas. The Katz-Klemm-Vafa conjecture for K3 surfaces. Preprint, arXiv:1404.6698

37. A. Vistoli. Intersection theory on algebraic stacks and on their moduli spaces. Invent. Math. 97 (1989), no. 3, 613-670.

Department of Mathematics and Research Institute of Mathematics, Seoul National University, Seoul 08826, Korea

E-mail address: kiem@snu.ac.kr 